\title{
Quantum criticality in the pseudogap Bose-Fermi Anderson and Kondo models: Interplay between fermion- and boson-induced Kondo destruction
}

\author{
J. H. Pixley, ${ }^{1}$ Stefan Kirchner, ${ }^{2,3}$ Kevin Ingersent, ${ }^{4}$ and Qimiao $\mathrm{Si}^{1}$ \\ ${ }^{1}$ Department of Physics and Astronomy, Rice University, Houston, Texas 77005, USA \\ ${ }^{2}$ Max Planck Institute for the Physics of Complex Systems, 01187 Dresden, Germany \\ ${ }^{3}$ Max Planck Institute for Chemical Physics of Solids, 01187 Dresden, Germany \\ ${ }^{4}$ Department of Physics, University of Florida, Gainesville, Florida 32611-8440, USA
}

(Received 10 June 2013; revised manuscript received 27 September 2013; published 10 December 2013)

\begin{abstract}
We address the phenomenon of critical Kondo destruction in pseudogap Bose-Fermi Anderson and Kondo quantum impurity models. These models describe a localized level coupled both to a fermionic bath having a density of states that vanishes like $|\epsilon|^{r}$ at the Fermi energy $(\epsilon=0)$ and, via one component of the impurity spin, to a bosonic bath having a sub-Ohmic spectral density proportional to $|\omega|^{s}$. Each bath is capable by itself of suppressing the Kondo effect at a continuous quantum phase transition. We study the interplay between these two mechanisms for Kondo destruction using continuous-time quantum Monte Carlo for the pseudogap Bose-Fermi Anderson model with $0<r<\frac{1}{2}$ and $\frac{1}{2} \leqslant s<1$, and applying the numerical renormalization group to the corresponding Kondo model. At particle-hole symmetry, the models exhibit a quantum-critical point between a Kondo (fermionic strong-coupling) phase and a localized (Kondo-destroyed) phase. The two solution methods, which are in good agreement in their domain of overlap, provide access to the many-body spectrum, as well as to correlation functions including, in particular, the single-particle Green's function and the static and dynamical local spin susceptibilities. The quantum-critical regime exhibits the hyperscaling of critical exponents and $\omega / T$ scaling in the dynamics that characterize an interacting critical point. The $(r, s)$ plane can be divided into three regions: one each in which the calculated critical properties are dominated by the bosonic bath alone or by the fermionic bath alone, and between these two regions, a third in which the bosonic bath governs the critical spin response but both baths influence the renormalization-group flow near the quantum-critical point.
\end{abstract}

DOI: 10.1103/PhysRevB.88.245111

PACS number(s): 71.10.Hf, 71.27.+a, 75.20.Hr

\section{INTRODUCTION}

Quantum criticality is currently being pursued in a number of strongly correlated materials ranging from intermetallic rare-earth compounds to insulating magnets and even engineered systems. This interest is spurred in part by the observation that unconventional superconductivity commonly occurs near quantum-critical points, as well as by mounting experimental evidence of the inadequacy of the traditional theoretical approach to continuous transitions at absolute temperature $T=0$. This approach, commonly referred to as the spin-density-wave or Hertz-Millis-Moryia picture, is based on an extension to zero temperature of Landau's theory of order-parameter fluctuations. ${ }^{1}$ The evidence for the breakdown of the spin-density-wave picture is particularly compelling in the context of intermetallic rare-earth compounds near an antiferromagnetic instability. As a result, a major thrust in the quest for a theoretical framework of critical phenomena beyond the Landau-Ginzburg-Wilson paradigm has centered around the question of how Kondo screening can be destroyed at a $T=0$ magnetic-ordering transition.

An extensively discussed alternative to the spin-densitywave picture is local quantum criticality, ${ }^{2,3}$ in which Kondo screening itself becomes critical precisely at the magneticordering transition. In the vicinity of such a Kondo-destruction quantum-critical point (QCP), scaling properties of the order parameter are very different from those predicted by spindensity-wave theory. For example, inelastic neutron-scattering experiments have shown that the dynamical spin (orderparameter) susceptibility exhibits frequency-over-temperature (or $\omega / T$ ) scaling, and displays a fractional exponent in the frequency and temperature dependencies over a wide range of momentum space. ${ }^{4}$ In addition, Hall measurements have provided evidence that the single-particle relaxation rate is linear in temperature, ${ }^{5}$ a behavior that arises naturally from $\omega / T$ scaling of the single-particle Green's function.

A microscopic model of local quantum criticality in heavyfermion metals has been provided ${ }^{3,6}$ through extended dynamical mean-field theory, which maps the Kondo lattice model to a single magnetic impurity coupled to both a fermionic conduction band and to one or more bosonic baths representing collective spin fluctuations. The critical point in the lattice model is then captured in terms of a critical destruction of the Kondo effect in the single-impurity problem. Such a construction gives rise to a QCP that is spatially local but has interacting critical modes in imaginary time, and that correctly captures $\omega / T$ scaling in the order-parameter susceptibility. While the full extended dynamical mean-field treatment simulates the lattice through self-consistent determination of the band and bath densities of states, valuable insight can be gained by studying Kondo-destruction QCPs in pure impurity problems.

This work investigates the quantum-critical destruction of the Kondo effect in pseudogap variants of the Ising-symmetric (or easy-axis) Bose-Fermi Anderson (BFA) and Bose-Fermi Kondo (BFK) models. In each model, a local degree of freedom couples to a band of conduction electrons having a density of states that vanishes as $|\epsilon|^{r}$ on approach to the Fermi energy $(\epsilon=0)$. The local degree of freedom is also coupled via the $z$ component of its spin to a bosonic bath having a density of states proportional to $\omega^{s}$ for frequencies up to some cutoff $\omega_{c}$ (i.e., for $0<\omega<\omega_{c}$ ). 
In the presence of a metallic conduction band (corresponding to an exponent $r=0$ ), the BFA and BFK models with a sub-Ohmic bosonic bath characterized by a bath exponent $0<s<1$ feature a second-order quantum phase transition ${ }^{7-14}$ (QPT) between a Kondo-screened (fermionic strong-coupling) phase and a localized (Kondo-destroyed) phase in which the bosons asymptotically suppress spin-flip scattering and a residual impurity moment survives to $T=0$. For $\frac{1}{2}<s<1$, the Ising-symmetry BFA and BFK models are thought ${ }^{1-14}$ to share the same critical properties as the corresponding sub-Ohmic spin-boson model, which features an interacting QCP characterized by critical exponents that vary continuously with the bath exponent $s$.

In the absence of the bosonic bath, by contrast, the pseudogap BFA and pseudogap BFK models reduce, respectively, to the pseudogap Anderson and pseudogap Kondo models, in which the depression of the low-energy density of states impedes the formation of a many-body Kondo screening cloud and gives rise to QPTs (Refs. 15-20) between a Kondo phase and a localized phase in which the impurity exhibits a free spin- $\frac{1}{2}$ at $T=0$. The QPTs in the pseudogap Anderson and Kondo models belong to the same universality class ${ }^{17-20}$ and are described by critical exponents that vary with the band exponent $r$. The pseudogap versions of the BFA and BFK models offer fascinating possibilities for nontrivial interplay between two different mechanisms for destruction of the Kondo effect, an interplay that we investigate in this work.

An SU(2)-symmetric version of the pseudogap BFK model (in which the Cartesian components of the impurity spin couple to different bosonic baths sharing the same exponent $s$ ) has been studied via perturbative renormalization-group (RG) methods, ${ }^{21,22}$ while the Ising-symmetric case of bosonic coupling to the $z$ component of the impurity spin has been the subject of preliminary investigation using the numerical renormalization group (NRG). ${ }^{23} \mathrm{~A}$ spinless variant of the model (coupling the impurity charge to a bosonic bath) has been addressed by perturbative and numerical RG techniques. ${ }^{24}$ In all these previous studies, the order-parameter susceptibility has been found to exhibit an anomalous $T^{-x}$ variation in the quantum-critical regime with an exponent $x=s$ independent of the band exponent $r$. One of the objectives of this work is to investigate more carefully the universality of this observation.

Another motivation for this study is to expand our understanding of the conditions that lead to $\omega / T$ or dynamical scaling of critical correlation functions near unconventional quantum criticality. It is well known that a quantum impurity model with a bulk component that is conformally invariant can be described by a boundary conformal field theory. ${ }^{25,26}$ In any such theory, a conformal mapping can be used to obtain correlators at temperatures $T>0$ from their $T=0$ counterparts. In particular, the zero-temperature two-point correlator of a primary conformal field $\Phi$ with scaling dimension $\lambda$ exhibits a power-law decay $\langle\Phi(\tau, T=0) \Phi(0, T=0)\rangle \sim \tau^{-2 \lambda}$. This gives rise, via a conformal mapping, to the scaling form ${ }^{27-29}$

$$
\langle\Phi(\tau, T) \Phi(0, T)\rangle \sim\left(\frac{\pi T}{\sin (\pi \tau T)}\right)^{2 \lambda} .
$$

The Fourier transform of Eq. (1) can be performed analytically and yields a function of $\omega_{n} / T$ that can be analytically continued to real frequencies provided ${ }^{30}$ that $2 \lambda<1$. Therefore, demonstration that a system obeys Eq. (1) with $2 \lambda<1$ is sufficient to show the presence of $\omega / T$ scaling, a characteristic feature of interacting QCPs.

In the pseudogap Anderson model, the conduction band density of states breaks conformal invariance, while in the metallic $(r=0)$ BFK model, the conduction band is conformally invariant but the bosonic bath is not. Nonetheless, local correlators of each model have been found ${ }^{20,31,32}$ to exhibit a boundary conformal scaling form in imaginary time, consistent with Eq. (1); such properties have been attributed to an enhanced symmetry at the QCPs. It is an interesting question as to whether the scaling form Eq. (1) also applies to the critical correlators of the pseudogap BFA and BFK models, where the densities of states of the fermionic and bosonic baths both break conformal invariance in the bulk.

We address these issues through a combination of techniques. For the particle-hole-symmetric pseudogap BFA model, we use a continuous-time quantum Monte Carlo (CT-QMC) method, ${ }^{33,34}$ which stochastically samples a perturbation expansion in the Anderson hybridization, to probe static and dynamical quantities. We also use the Bose-Fermi extension of the NRG method $^{13,14}$ to resolve the critical spectrum and extract static critical exponents of the pseudogap BFK model. Our results show that the two models are in the same universality class.

We find that within different ranges of the exponents $r$ and $s$, the measured critical exponents are determined by the fermionic band alone, by the bosonic bath alone, or by both the fermions and the bosons. We show that both the single-particle Green's function and the local spin susceptibility obey the scaling form of Eq. (1) with exponents $2 \lambda<1$, proving that each correlator obeys $\omega / T$ scaling in both the quantum coherent $(\omega>T)$ and the relaxational $(\omega<T)$ regimes. Agreement with NRG results for static quantities confirms the ability of the CT-QMC approach to study quantum-critical properties of models involving bosons.

The remainder of the paper is organized as follows: Section II introduces the pseudogap Bose-Fermi models and Sec. III briefly describes the numerical methods used to solve these models. An overview of the phase diagram in Sec. IV is followed in Sec. V by a detailed description of the quantum-critical properties. These results are summarized and discussed in Sec. VI.

\section{MODELS}

The Bose-Fermi Anderson impurity model with Isingsymmetric bosonic coupling is described by the Hamiltonian

$$
\begin{aligned}
H_{\mathrm{BFA}}= & \sum_{\mathbf{k} \sigma} \epsilon_{\mathbf{k}} c_{\mathbf{k} \sigma}^{\dagger} c_{\mathbf{k} \sigma}+\epsilon_{d}\left(n_{d \uparrow}+n_{d \downarrow}\right)+U n_{d \uparrow} n_{d \downarrow} \\
& +\frac{V}{\sqrt{N_{k}}} \sum_{\mathbf{k}, \sigma}\left(d_{\sigma}^{\dagger} c_{\mathbf{k} \sigma}+c_{\mathbf{k} \sigma}^{\dagger} d_{\sigma}\right) \\
& +\sum_{q} \omega_{q} \phi_{q}^{\dagger} \phi_{q}+\frac{1}{2} g\left(n_{d \uparrow}-n_{d \downarrow}\right) \sum_{q}\left(\phi_{q}^{\dagger}+\phi_{-q}\right),
\end{aligned}
$$


where $c_{\mathbf{k} \sigma}$ annihilates a conduction band electron with wave vector $\mathbf{k}$, energy $\epsilon_{\mathbf{k}}$, and spin $z$ component $\frac{1}{2} \sigma$ with $\sigma=1$ (or $\uparrow$ ) or -1 (or $\downarrow$ ); $d_{\sigma}$ annihilates an impurity electron with energy $\epsilon_{d}$ and spin $z$ component $\frac{1}{2} \sigma ; n_{d \sigma}=d_{\sigma}^{\dagger} d_{\sigma} ; \phi_{q}$ annihilates a boson of energy $\omega_{q}$; and $N_{k}$ is the number of unit cells in the host (i.e., the number of distinct $\mathbf{k}$ points). The other energy scales entering Eq. (2) are the Coulomb repulsion $U$ between two electrons in the impurity level, the local hybridization $V$ between the impurity level and the conduction band, and the coupling $g$ between the $z$ component of the impurity spin and the bosonic bath. We focus in this paper on cases $\epsilon_{d}=-\frac{1}{2} U$ corresponding to particle-hole-symmetric impurities, but briefly discuss the effect of breaking this symmetry in Sec. VD.

Over a wide region of its parameter space, the low-energy properties of $H_{\mathrm{BFA}}$ can be mapped ${ }^{34}$ via a Schrieffer-Wolff transformation onto the Ising-symmetry Bose-Fermi Kondo Hamiltonian

$$
\begin{aligned}
H_{\mathrm{BFK}}= & \sum_{\mathbf{k} \sigma} \epsilon_{\mathbf{k}} c_{\mathbf{k} \sigma}^{\dagger} c_{\mathbf{k} \sigma}+J \mathbf{S} \cdot \mathbf{s}_{c}+\frac{W}{N} \sum_{\mathbf{k}, \mathbf{k}^{\prime} \sigma} c_{\mathbf{k} \sigma}^{\dagger} c_{\mathbf{k}^{\prime} \sigma} \\
& +\sum_{q} \omega_{q} \phi_{q}^{\dagger} \phi_{q}+g S_{z} \sum_{q}\left(\phi_{q}^{\dagger}+\phi_{-q}\right),
\end{aligned}
$$

where $J$ is the Kondo coupling, $W$ parametrizes nonmagnetic potential scattering, and

$$
\mathbf{s}_{c}=\frac{1}{2 N_{k}} \sum_{\mathbf{k}, \mathbf{k}^{\prime} \sigma, \sigma^{\prime}} c_{\mathbf{k} \sigma}^{\dagger} \boldsymbol{\sigma}_{\sigma \sigma^{\prime}} c_{\mathbf{k}^{\prime} \sigma^{\prime}}
$$

is the onsite conduction band spin, with $\sigma$ being a vector of Pauli spin matrices. For $\epsilon_{d}=-\frac{1}{2} U$, the potential scattering vanishes for electrons on the Fermi surface, and we can set $W=0$. Although the bare Kondo exchange term is SU(2) symmetric, the bosonic coupling breaks spin rotational invariance. As a result, the RG description of the BFK model in terms of renormalized couplings requires consideration of an anisotropic exchange $J_{z} S_{z} s_{c, z}+\frac{1}{2} J_{\perp}\left(S^{+} s_{c}^{-}+S^{-} s_{c}^{+}\right)$.

For both models, we assume a conduction band (fermionicbath) density of states

$$
\rho_{F}(\epsilon)=N^{-1} \sum_{\mathbf{k}} \delta\left(\epsilon-\epsilon_{\mathbf{k}}\right)=\rho_{0}|\epsilon / D|^{r} \Theta(D-|\epsilon|)
$$

with a power-law pseudogap described by $0<r<\frac{1}{2}$, and a sub-Ohmic bosonic bath specified by

$$
\rho_{B}(\omega)=\sum_{q} \delta\left(\omega-\omega_{q}\right)=K_{0}^{2} \omega_{c}^{1-s} \omega^{s} \Theta(\omega) \Theta\left(\omega_{c}-\omega\right)
$$

with $\frac{1}{2} \leqslant s<1$. The pseudogap density of states $\rho_{F}(\epsilon)$ leads to a BFA model with the hybridization function $\Gamma_{F}(\epsilon)=$ $\pi V^{2} \rho_{F}(\epsilon)=\Gamma|\epsilon / D|^{r} \Theta(D-|\epsilon|)$, where $\Gamma=\pi \rho_{0} V^{2}$.

Various limiting cases of Eqs. (2) and (3) have been studied previously. For $g=0$, the pseudogap Bose-Fermi models simplify to their pure-fermionic counterparts, in which a pseudogap critical point separates Kondo and free-moment phases; at particle-hole symmetry, this critical point exists only for $0<r<\frac{1}{2}$ (Ref. 17). In the absence of the conduction band, $H_{\mathrm{BFA}}$ and $H_{\mathrm{BFK}}$ both reduce to the sub-Ohmic spin-boson model in zero transverse field, which has two degenerate ground states in which the bosonic coupling localizes the impurity either in its up- or down-spin configuration. For $r=0$, the pseudogap BFA and BFK models reduce to their metallic counterparts where the critical properties for Ising symmetry are thought ${ }^{11-14}$ to coincide with those of the spin-boson model for $\frac{1}{2} \leqslant s<1$. In the sections that follow, we show that the quantum criticality in the full pseudogap Bose-Fermi models described by Eqs. (2) and (3) falls into one of three distinct types, depending on the values of the bath exponents $r$ and $s$, with one of these types being governed by a mixed Bose-Fermi QCP unlike those seen in any of the limiting cases.

\section{METHODS}

\section{A. Continuous-time quantum Monte Carlo}

This work uses a form of the CT-QMC method described in Ref. 34. To make $H_{\mathrm{BFA}}$ suitable for application of the approach, we apply a canonical transformation to eliminate the term linear in bosonic operators. This is achieved by the generator $S=\frac{1}{2} g\left(n_{d \uparrow}-n_{d \downarrow}\right) \sum_{q} \omega_{q}^{-1}\left(\phi_{q}^{\dagger}-\phi_{-q}\right)$, which transforms Eq. (2) to

$$
\begin{aligned}
\tilde{H}_{\mathrm{BFA}}= & e^{S} H_{\mathrm{BFA}} e^{-S}=\sum_{\mathbf{k} \sigma} \epsilon_{\mathbf{k}} c_{\mathbf{k} \sigma}^{\dagger} c_{\mathbf{k} \sigma}+\tilde{\epsilon}_{d}\left(\tilde{n}_{\uparrow}+\tilde{n}_{\downarrow}\right)+\tilde{U} \tilde{n}_{\uparrow} \tilde{n}_{\downarrow} \\
& +\frac{V}{\sqrt{N_{k}}} \sum_{\mathbf{k} \sigma}\left(\tilde{d}_{\sigma}^{\dagger} c_{\mathbf{k} \sigma}+c_{\mathbf{k} \sigma}^{\dagger} \tilde{d}_{\sigma}\right)+\sum_{q} \omega_{q} \phi_{q}^{\dagger} \phi_{q},
\end{aligned}
$$

where $\quad \tilde{d}_{\sigma}^{\dagger}=d_{\sigma}^{\dagger} \exp \left[\frac{1}{2} \sigma g \sum_{q} \omega_{q}^{-1}\left(\phi_{q}^{\dagger}-\phi_{-q}\right)\right], \quad \tilde{U}=$ $U+\frac{1}{2} g^{2} \sum_{q} \omega_{q}^{-1}$, and $\tilde{\epsilon}_{d}=-\frac{1}{2} \tilde{U}$. Physically, the canonical transformation can be viewed as dressing the impurity with a bosonic cloud, which in turn renormalizes $U, \epsilon_{d}, d_{\sigma}$, and $d_{\sigma}^{\dagger}$ without breaking particle-hole symmetry.

We are now in a position to calculate the partition function by expanding in the hybridization $V$. This is similar to the approach in Refs. 35 and 36, except that we couple the bosonic bath to the $z$ component of the impurity's spin rather than to its occupancy. [An extension of this method to the case of an SU(2)-symmetric spin-boson coupling has recently been proposed. ${ }^{37}$ ] The resulting perturbation expansion is then sampled stochastically using a Metropolis algorithm. Tracing out the fermionic band, which is unchanged by the presence of bosons, ${ }^{33}$ allows the partition function to be recast as $Z=\sum_{k} \int D[k] W_{k}$, where at perturbation order $k$ there are $k$ segments along the imaginary time axis, each one defined by a pair of renormalized impurity operators $\tilde{d}_{\sigma_{j}^{\prime}}\left(\tau_{j}^{\prime}\right)$ and $\tilde{d}_{\sigma_{j}^{\prime}}^{\dagger}\left(\tau_{j}^{\prime \prime}\right)$. The weight of a particular configuration of segments is $W_{k}=w_{F} w_{\text {loc }} w_{B}$, where $w_{F}$ is the weight of the band fermions, $w_{\text {loc }}$ is the weight of the local configuration, and $w_{B}$ is the weight of the bosonic bath. $w_{F}$ can be obtained as a product of determinants and $w_{\text {loc }}$ is calculated in terms of the length and overlap of imaginary-time segments (for details, see Ref. 33). If the operators in a given segment configuration are time ordered, with $s_{i}=1$ $(-1)$ indicating that the $i$ th operator acts at time $\tau_{i}$ to create (annihilate) an electron of spin $z$ component $\sigma_{i}$, then the bosonic weight is $w_{B}=\left\langle e^{\frac{1}{2} g s_{2 k} \sigma_{2 k} \hat{B}\left(\tau_{2 k}\right)} \ldots e^{\frac{1}{2} g s_{1} \sigma_{1} \hat{B}\left(\tau_{1}\right)}\right\rangle$, where $\hat{B}(\tau)=\sum_{q} \omega_{q}^{-1}\left[\phi_{q}^{\dagger}(\tau)-\phi_{-q}(\tau)\right]$. Tracing out the bosons and 
performing the momentum summation yields

$$
w_{B}=\exp \left\{-\frac{g^{2}}{4}\left[k B(0)+\sum_{1 \leqslant i<j \leqslant 2 k} s_{i} s_{j} \sigma_{i} \sigma_{j} B\left(\tau_{j}-\tau_{i}\right)\right]\right\} \text {. }
$$

To calculate the function $B(\tau)$ we replace the hard cutoff of the bosonic spectral function in Eq. (6) by an exponential cutoff $\rho_{B}(\omega) \rightarrow K_{0}^{2} \omega_{c}^{1-s} \omega^{s} \Theta(\omega) \exp \left(-\omega / \omega_{c}\right)$, which yields

$$
\begin{aligned}
B(\tau)= & K_{0}^{2}\left(\omega_{c} / T\right)^{1-s} \Gamma(s-1)\left[\zeta\left(s-1, \tau T+T / \omega_{c}\right)\right. \\
& \left.+\zeta\left(s-1,1-\tau T+T / \omega_{c}\right)\right],
\end{aligned}
$$

where $\Gamma(x)$ is the gamma function and $\zeta(t, z)$ is the Hurwitz zeta function. ${ }^{38}$ Now, the local update procedure is identical to that in Ref. 35. In all the CT-QMC calculations reported below, we have considered the low-temperature scaling limit $\left(T \ll \omega_{c}\right)$ and consequently dropped the terms $T / \omega_{c}$ from Eq. (9). We have checked that retaining or discarding these terms does not change any of the universal features at the QCP such as critical exponents.

We perform an unbiased CT-QMC study of the quantumcritical properties of the pseudogap BFA model by applying finite-temperature scaling to determine the location of the QCP. We measure powers of the local magnetization

$$
\left\langle M_{z}^{n}\right\rangle=\left\langle\left[T \int_{0}^{1 / T} d \tau S_{z}(\tau)\right]^{n}\right\rangle
$$

where $S_{z}(\tau)=\frac{1}{2}\left[n_{d \uparrow}(\tau)-n_{d \downarrow}(\tau)\right]$, from which the Binder cumulant

$$
U_{4}(T, g)=\frac{\left\langle M_{z}^{4}\right\rangle}{\left\langle M_{z}^{2}\right\rangle^{2}}
$$

is obtained. Similar to the case without bosons ${ }^{20,32}$ we find that swap moves ${ }^{33}$ are essential to accurately calculate the Binder cumulant. Near classical phase transitions, the Binder cumulant is a function of system size and temperature, and finite-size scaling allows one to obtain the critical temperature in the thermodynamic limit from classical Monte Carlo simulations of finite systems. ${ }^{39}$ For the current quantum-mechanical problem, the inverse temperature $1 / T$ and coupling $g$ play the roles of system size and temperature, respectively, so taking the zero-temperature limit allows one to determine the critical coupling $g_{c}$. In the vicinity of the critical point, the Binder cumulant obeys the scaling form ${ }^{39}$

$$
U_{4}(T, g)=\tilde{U}_{4}\left(\frac{g / g_{c}-1}{T^{1 / \nu}}\right),
$$

where $v$ is the correlation-length exponent defined in Eq. (17). Therefore, finite-temperature scaling applied to the Binder cumulant can be used to extract the correlation-length critical exponent.

We also use CT-QMC to measure the single-particle Green's function $G_{\sigma}(\tau, T)=\left\langle T_{\tau} d_{\sigma}(\tau) d_{\sigma}^{\dagger}(0)\right\rangle[\equiv G(\tau, T)$ in zero magnetic field] and the local spin susceptibility $\chi_{\text {loc }}(\tau, T)=\left\langle T_{\tau} S_{z}(\tau) S_{z}(0)\right\rangle$, as described in Ref. 33. For the susceptibility calculations presented in this paper, we have used the segment representation, and have checked that the results are consistent with those calculated using a "matrix" formalism. ${ }^{33}$ The static local spin susceptibility can then be determined as $\chi_{\text {stat }}(T)=\chi_{\text {loc }}(\omega=0, T)=\int_{0}^{1 / T} d \tau \chi_{\mathrm{loc}}(\tau, T)$, where we have set the Landé $g$ factor and the Bohr magneton to unity. For the noninteracting problem $(U=g=0)$, with $r=$ 0.4 , we find agreement within numerical accuracy between the exact and CT-QMC results for both the Green's function and the local spin susceptibility. ${ }^{34}$

The CT-QMC results reported in the following were obtained for $r=0.4, \omega_{c}=K_{0}^{-1}=D, \Gamma=0.1 D, U=0.01 D$, and for two different bath exponents: $s=0.6$ and 0.8 . It is known from previous work ${ }^{20}$ that in the absence of bosons, the QCP point for $r=0.4$ and $\Gamma=0.1 D$ occurs at $U_{c} \simeq$ $0.085 D$. Therefore, by fixing $U=0.01 D$ and adjusting the bosonic coupling $g$, we are able to disentangle the pseudogap Bose-Fermi QCP from the pure-fermionic pseudogap QCP.

\section{B. Numerical renormalization group}

The Bose-Fermi NRG (Refs. 13 and 14) treatment of the Hamiltonian (3) entails three key steps: (i) Partition of the fermionic and bosonic baths described by $\rho_{F}(\epsilon)$ and $\rho_{B}(\omega)$ into logarithmic bins spanning the energy ranges $\Lambda^{-j}<$ $|\epsilon| / D, \omega / \omega_{c} \leqslant \Lambda^{-(j-1)}$, where $j=1,2,3, \ldots$ and $\Lambda>1$ is the Wilson discretization parameter. Within each logarithmic bin, the continuum is replaced by a single state, namely, the linear combination of states that couples to the impurity. (ii) Tridiagonalization of the baths, yielding the Hamiltonian

$$
\begin{aligned}
H_{\mathrm{BFK}}= & D \sum_{n=0}^{\infty} \sum_{\sigma}\left[\epsilon_{n} f_{n \sigma}^{\dagger} f_{n \sigma}+\tau_{n}\left(f_{n \sigma}^{\dagger} f_{n-1, \sigma}+\text { H.c }\right)\right] \\
& +\omega_{c} \sum_{m=0}^{\infty}\left[e_{m} b_{m}^{\dagger} b_{m}+t_{m}\left(b_{m}^{\dagger} b_{m-1}+\text { H.c }\right)\right] \\
& +F^{2} \rho_{0} J \vec{S} \cdot \sum_{\sigma, \sigma^{\prime}} f_{0 \sigma}^{\dagger} \vec{\sigma}_{\sigma \sigma^{\prime}} f_{0 \sigma^{\prime}}+B K_{0} g S_{z}\left(b_{0}+b_{0}^{\dagger}\right) .
\end{aligned}
$$

All information about $\rho_{F}(\epsilon)$ is encoded in $D$ and the dimensionless coefficients $F$ and $\left\{\epsilon_{n}, \tau_{n}\right\}$, while $\rho_{B}(\omega)$ is parametrized by $\omega_{c}$ and the dimensionless quantities $B$ and $\left\{e_{m}, t_{m}\right\}$. For a particle-hole symmetric $\rho_{F}(\epsilon)$ such as that in Eq. (5), $\epsilon_{n}=0$ for all $n$. For large values of $n$, the remaining tight-binding coefficients satisfy $\tau_{n} \sim D \Lambda^{-n / 2}$ and $\left|e_{m}\right|, t_{m} \sim$ $\omega_{c} \Lambda^{-m}$. (iii) Iterative solution of Eq. (13) on fermionic chains restricted to sites $0 \leqslant n \leqslant N$ with $N=0,1,2, \ldots$. Due to the faster decay of the bosonic hopping coefficients with increasing index $m$, the bosonic chain is restricted to $0 \leqslant m \leqslant N / 2$, meaning that a site is added to this chain only at even values of $N$. As in the conventional (pure-fermionic) $\mathrm{NRG}$, the $N_{s}$ many-body states of lowest energy are retained to form the basis for iteration $N+1$. In problems involving bosonic baths, it is also necessary to truncate the Fock space on each site of the bosonic chain. In this work, we employed a basis of boson number eigenstates $0 \leqslant b_{m}^{\dagger} b_{m} \leqslant N_{b}$.

The NRG calculation of critical exponents in the vicinity of a QCP is carried out as described previously for the metallic $(r=0)$ BFK model ${ }^{13,14}$ and as illustrated in a preliminary publication on the pseudogap BFK model. ${ }^{23}$ The local magnetization (equivalent to $\left\langle M_{z}\right\rangle$ above) and the static 
local susceptibility are evaluated as

$$
M_{\mathrm{loc}}=\left\langle S_{z}\right\rangle, \quad \chi_{\mathrm{stat}}=\partial M_{\mathrm{loc}} / \partial h_{\mathrm{loc}},
$$

where $h_{\text {loc }}$ is a magnetic field that couples only to the impurity through an additional Hamiltonian term $h_{\mathrm{loc}} S_{z}$. All results reported below were obtained for $\omega_{c}=\sqrt{\pi} / K_{0}=$ $D=\rho_{0}^{-1}=1$, using Wilson discretization parameter $\Lambda=9$ and a bosonic truncation parameter $N_{b}=8$, and retaining after each iteration $N_{s}=500$ many-body multiplets corresponding to approximately 900 eigenstates. ${ }^{40}$ Experience from previous studies ${ }^{13,14,23}$ indicates that critical exponents calculated for these NRG parameter choices are well converged with respect to discretization errors (induced by the departure of $\Lambda$ from its continuum value 1) and truncation errors (arising from restricting the values of $N_{s}$ and $N_{b}$ ).

Over the range of bosonic exponents $0<s<1$, the BoseFermi NRG yields critical exponents for the case $r=0$ that reproduce those obtained via NRG (Ref. 41) for the spin-boson model having the same exponent $s$. It has been suggested in Refs. 42 and 43 that, for $0<s<\frac{1}{2}$, errors associated with the NRG calculation on a finite bosonic Wilson chain lead to unphysical results with nontrivial exponents and hyperscaling. On the other hand, for this same range of $s$, Refs. 44-46 have demonstrated an $\omega / T$ scaling for both the leading and subleading components of the self-energy, which provides evidence for the interacting nature of the fixed point. Since this issue has yet to be fully resolved, we restrict ourselves in this paper to study of the range $\frac{1}{2} \leqslant s<1$.

\section{PHASES}

In this section, we describe the two zero-temperature phases of the pseudogap BFA and BFK models that can be accessed by tuning one model parameter while holding all other parameters constant, as illustrated in the case of the BFK model by the horizontal and vertical dashed lines in Fig. 1. We will assume for the moment that the bosonic coupling $g$ is the parameter that is varied. It is important to emphasize that, if carried out at any temperature $T>0$, such a variation invariably produces smooth crossovers in physical properties, represented schematically by the crossing of dashed lines on the $T$ versus $g$ diagram in Fig. 2. Only at $T=0$ is it possible to drive the pseudogap BFA or BFK model through a QPT at $g=g_{c}$ separating a Kondo phase (reached for $g<g_{c}$ ) from a localized phase (accessed for $g>g_{c}$ ).

The Kondo phase can be characterized by the vanishing (as $T \rightarrow 0$ ) of the long-imaginary-time value of the local spin susceptibility $\chi_{\operatorname{loc}}(\tau=1 / 2 T, T)$. Correspondingly, the static local susceptibility $\chi_{\text {stat }}(T)$ approaches a constant at low temperatures (see, for example, the squares in Figs. 5 and 9), signaling that the impurity spin is screened. Since these behaviors (and all other universal low-energy properties found for $g<g_{c}$ ) prove to be identical to those in the Kondo phase of the pseudogap Anderson and Kondo models, and independent of the bosonic bath exponent $s$, we associate them with a RG fixed point located at renormalized couplings $g=0$ and either $\Gamma=\infty$ or $J=\infty$.

In the localized phase, by contrast, $\chi_{\text {loc }}(\tau=1 / 2 T, T)$ approaches a constant $C(g)>0$ in the limit $T \rightarrow 0$ and the static local susceptibility assumes the Curie-law form

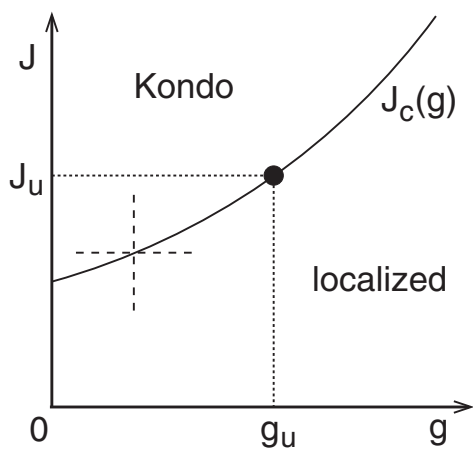

FIG. 1. Schematic zero-temperature phase diagram for the pseudogap BFK model on the plane spanned by the bosonic coupling $g$ and the Kondo exchange coupling $J$. The system undergoes a QPT at any crossing of the solid line $J=J_{c}(g)$ representing the boundary between the two stable phases: Kondo and localized. In this work, we consider crossings achieved by varying just one model parameter, as exemplified by the horizontal and vertical dashed lines. The labels $g_{u}$ and $J_{u} \equiv J_{c}\left(g_{u}\right)$ mark the point along the phase boundary at which the temperature $T_{u}$, the upper limit of the quantum-critical regime (see Fig. 2), takes its maximum value. The terminus of the phase boundary at $g=0, J=J_{c}(0)$ corresponds to the QPT of the pure-fermionic Kondo model. The pseudogap BFA model has a very similar phase diagram on the $g-\Gamma$ plane at any fixed, positive value of $U=-2 \epsilon_{d}$.

$\chi_{\text {stat }}(T)=C(g) / T$ (triangles in Figs. 5 and 9) characteristic of a free spin whose size is $\propto \sqrt{C(g)}$. In this low-temperature regime, the impurity spin is essentially decoupled from the conduction band and follows the fluctuations of the bosonic bath. The asymptotic low-energy properties are governed by a RG fixed point located at renormalized couplings $g=\infty$ and either $\Gamma=0$ or $J=0$.

The two phases described above are separated by a QPT occurring at bosonic coupling $g_{c}$ where the Curie constant $C(g)$ extrapolates continuously to zero as $g$ is decreased. As a result, $\lim _{T \rightarrow 0} T \chi_{\text {stat }}$ can serve as an order parameter for the QPT, ${ }^{17,18}$ vanishing throughout the Kondo phase and equaling the Curie constant in the localized phase. However,

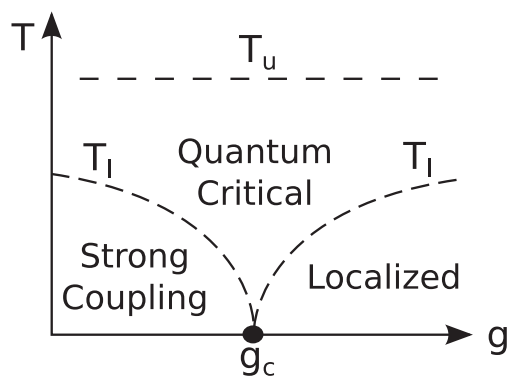

FIG. 2. Schematic $T$ vs $g$ diagram in the vicinity of the pseudogap Bose-Fermi QCP studied in this work. The QCP dominates the physics in a temperature window between crossover scales $T_{u}$ and $T_{l}$ (dashed lines). For $g<g_{c}$ and $0<T \lesssim T_{l}$, the system is in its Kondo regime, whereas for $g>g_{c}$ and $0<T \lesssim T_{l}$, the spin becomes decoupled from the conduction band and the system is in the localized regime. At $T=0$ (only), cases $g<g_{c}$ and $g>g_{c}$ correspond to different phases, distinguished by the value of the order parameter $\psi$ defined in Eq. (15). 
it is conventional instead to take as the order parameter

$$
\psi=\lim _{h_{\mathrm{loc}} \rightarrow 0^{-}} M_{\mathrm{loc}}(T=0),
$$

which rises continuously from zero on entry into the localized phase.

If one fixes all parameters apart from $\Gamma$ and $g$ in the BFA model ( $J$ and $g$ in the BFK model), then the function $g_{c}(\Gamma)$ $\left[g_{c}(J)\right]$ defines the boundary between the Kondo and localized phases. This boundary, shown for the Kondo case as the solid line in Fig. 1, is anchored at $g=0$ by the QCP of the purefermionic pseudogap models. One of the central questions addressed in our work is whether the critical behavior reached by crossing the phase boundary at $g>0$ coincides with or differs from that for $g=0$.

Our two numerical techniques lend themselves to different approaches for locating the phase boundary. In the CT-QMC treatment of the pseudogap BFA model, the low-temperature limit of the Binder cumulant $U_{4}$ evolves continuously from $U_{4}=3$ in the Kondo phase to $U_{4}=1$ in the localized phase. $U_{4}$ is independent of temperature at the critical bosonic coupling $g_{c}$, but not at other nearby values of $g$. Thus, one can find $g_{c}$ via the intersection of curves $U_{4}$ versus $g$ for different (low) temperatures (see Figs. 3 and 7).

Within the NRG, one can identify the phase boundary of the pseudogap BFK model through examination of the asymptotic low-energy many-body spectrum, the $T \rightarrow 0$ values of thermodynamic properties such as the impurity contribution to the entropy ( $S_{\mathrm{imp}}=2 r \ln 2$ in the Kondo phase, and $S_{\mathrm{imp}}=\ln 2$ in the localized phase), or the static local spin susceptibility $\chi_{\text {stat }}(T)$. In the present study of the pseudogap BFK model, rather than calculating $g_{c}$ as a function of $J$, we have instead determined the critical Kondo coupling $J_{c}$ for different values of $g$. For given bath exponents $r$ and $s$, points on the phase boundary at any $g>0$ are all found to share the same many-body spectrum and the same power laws in the quantum-critical regime $0 \leqslant T \lesssim T_{u}$ (see Fig. 2). This universality strongly suggests that the quantum criticality is governed by a single QCP to which the system flows starting from any point on the curve $J_{c}(g)$. However, the upper temperature $T_{u}$, which marks the energy scale at which

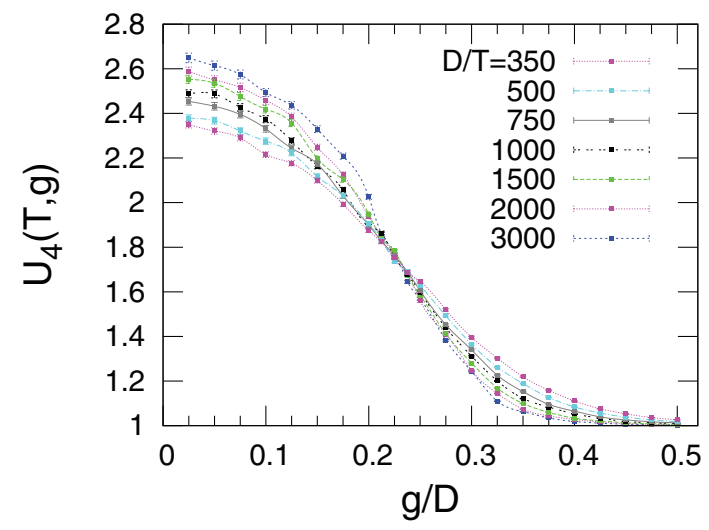

FIG. 3. (Color online) Binder cumulant $U_{4}$ vs bosonic coupling $g$ for $r=0.4$ and $s=0.6$ at different inverse temperatures $\beta=1 / T$. There is a clear intersection of all curves at the critical coupling $g_{c} / D=0.225(7)$. Error bars for Binder cumulant calculations are obtained from a jackknife error analysis.
RG flow first brings the system under the influence of the QCP, varies widely along the boundary. In order to provide the most accurate possible account of the quantum-critical properties, we focus in the following on NRG results obtained for the boundary point $g=g_{u}, J=J_{u} \equiv J_{c}\left(g_{u}\right)$ that yields the highest value of $T_{u}$ for given $(r, s)$, and can therefore be assumed to lie closest to the QCP. An advantage of this approach is that for certain $(r, s)$ pairs, we find $g_{u}=0$, making clear that in such cases the QCP is of pure-fermionic character.

In what follows, we write $\Delta=g-g_{c}$ for the BFA model and $\Delta=J_{c}-J$ for the BFK model to denote the system's distance from the phase boundary, with $\Delta<0$ describing the Kondo phase and $\Delta>0$ describing the localized phase.

\section{RESULTS NEAR THE QUANTUM-CRITICAL POINT}

\section{A. Static critical behavior}

At any point lying on the phase boundary between the Kondo and localized phases, the static susceptibility exhibits a temperature dependence

$$
\chi_{\text {stat }}(T ; \Delta=0) \simeq A T^{-x(r, s)}
$$

for all temperatures in the quantum-critical window $0<$ $T \lesssim T_{u}$. Here, the exponent $x$ is a universal property of the pseudogap Bose-Fermi QCP (i.e., $x$ depends only on the bath exponents $r$ and $s$ ), whereas the crossover temperature $T_{u}$ and the prefactor $A$ can vary from point to point along the phase boundary; as noted above, $T_{u}$ is highest for the boundary point that lies nearest to the QCP.

Close to but not precisely on the phase boundary, the system enters the quantum-critical regime once the temperature drops below roughly the same scale $T_{u}$ found for $\Delta=0$. However, further lowering the temperature produces a second crossover to an asymptotic regime $T \lesssim T_{l}$ governed either by the Kondo fixed point (for $\Delta<0$ ) or by the localized fixed point (for $\Delta>0$ ) (see Fig. 2). Unlike $T_{u}$, the lower crossover scale $T_{l}$ shows significant $\Delta$ dependence, and vanishes continuously upon approach to the phase boundary according to

$$
T_{l} \propto|\Delta|^{v},
$$

where $v$ is the correlation-length exponent.

In the absence of bosons, the exponent $x(r, s)$ necessarily assumes the value $x_{F}(r)$ found at the QCP of the pure-fermionic pseudogap Anderson and Kondo models. With a constant fermionic density of states (i.e., $r=0$ ) and isotropic, $X Y$, or Ising symmetry of the bosonic couplings, it is known ${ }^{11,12}$ that $x(0, s)$ for $\frac{1}{2}<s<1$ reduces to the exponent $x_{B}(s)=s$ of the spin-boson model. Based on perturbative RG, ${ }^{21,22}$ it has been concluded that $x(r, s)$ for a spin-isotropic version of the pseudogap BFK model is independent of $r$, an observation that agrees with asymptotically exact results obtained in the dynamical large- $N$ limit ${ }^{47,48}$ where the symmetry group of the spin-isotropic pseudogap BFK model is generalized from $\mathrm{SU}(2)$ to $\mathrm{SU}(N) .{ }^{49}$ A preliminary $\mathrm{NRG}$ study ${ }^{23}$ of the easy-axis pseudogap BFK model also found $x(r, s)=s$. However, that study considered only exponent pairs $(r, s)$ for which $x_{B}(s)<$ $x_{F}(r)$, a regime in which it is quite plausible that the bosons should dominate the singular part of the spin response. In this study, we have also investigated cases where $x_{F}(r)<x_{B}(s)$ 
that offer better prospects for finding fermion-dominated spin dynamics.

In what follows, we show using both CT-QMC calculations and the NRG that the static magnetic critical exponent $x$ is simply the smaller of the exponents governing the cases of pure-fermionic and pure-bosonic critical Kondo destruction:

$$
x(r, s)=\min \left[x_{F}(r), x_{B}(s)\right] .
$$

We focus primarily on the pseudogap exponent $r=0.4$ and two values of the bath exponent: $s=0.6$ and 0.8 . For the pure-fermionic Kondo model, the critical susceptibility is described by a temperature exponent $x_{F}(r=0.4)=0.688(1)$ obtained within the NRG, ${ }^{18}$ consistent with the value $x_{F}=$ 0.68(3) found using CT-QMC for the corresponding Anderson model. ${ }^{20}$ (Here and throughout the remainder of the paper, a number in parentheses indicates our estimated uncertainty in the last digit.) For the Bose-Fermi models, therefore, the cases $(r, s)=(0.4,0.6)$ and $(0.4,0.8)$ are representative of the regimes $x_{B}<x_{F}$ and $x_{B}>x_{F}$, respectively. For each of these cases, we present CT-QMC results for the pseudogap BFA model and NRG results for the pseudogap BFK model, finding the critical behavior of the two models to be fully equivalent.

We supplement the detailed results for $r=0.4, s=0.6$ and 0.8 with static critical exponents for a larger set of $(r, s)$ pairs as obtained for the BFK model using the NRG. In addition to $x$ and $v$ introduced in Eqs. (16) and (17), we consider exponents $\beta$ and $\delta$ defined via the relations ${ }^{14}$

$$
\begin{gathered}
M_{\mathrm{loc}}\left(\Delta>0 ; T=0, h_{\mathrm{loc}} \rightarrow 0\right) \propto \Delta^{\beta}, \\
M_{\mathrm{loc}}\left(h_{\mathrm{loc}} ; \Delta=0, T=0\right) \propto\left|h_{\mathrm{loc}}\right|^{1 / \delta} .
\end{gathered}
$$

If the QPT occurs below its upper critical dimension, yielding an interacting QCP, one expects the singular component of the free energy to take the form

$$
F_{\text {crit }}=T f\left(\frac{|\Delta|}{T^{1 / v}}, \frac{\left|h_{\text {loc }}\right|}{T^{(1+x) / 2}}\right),
$$

where $f$ is a scaling function. With this ansatz, the exponents $\beta$ and $\delta$ are related to $\nu$ and $x$ by hyperscaling relations

$$
\begin{gathered}
\beta=v(1-x) / 2, \\
\delta=(1+x) /(1-x) .
\end{gathered}
$$

In each of the cases we have studied, the value of $x$ is consistent with Eq. (18), and wherever we have tested them, the hyperscaling Eqs. (22) and (23) are well obeyed. Based on the value of $v(r, s)$ [or alternatively, $\beta(r, s)$ ], as well as an analysis of the many-body spectrum at the QCP, we are led to subdivide the region of the $(r, s)$ plane in which $x(r, s)=x_{B}(s)$ into two parts: one in which the fermions appear to play no role in the critical behavior, and another in which bosonic and fermionic fluctuations combine to produce critical behavior unlike that found in either the metallic $(r=0)$ BFK model or the pseudogap Kondo model.

\section{Results for $r=0.4, s=0.6$}

Figure 3 shows the variation with bosonic coupling $g$ of the Binder cumulant $U_{4}$ of the pseudogap BFA model for $r=0.4, s=0.6$ at different temperatures as calculated using

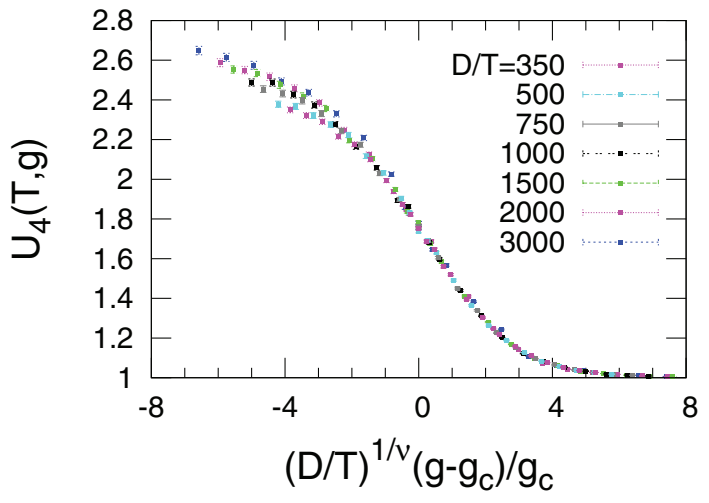

FIG. 4. (Color online) Data collapse of the Binder cumulant $U_{4}$ for $r=0.4$ and $s=0.6$ using Eq. (12). An inverse correlation-length exponent $v(r=0.4, s=0.6)^{-1}=0.25(3)$ produces an excellent collapse of data in the vicinity of the critical point.

CT-QMC. The intersection of the curves places the QCP at $g_{c} / D=0.225(7)$.

The scaling form (12) of the Binder cumulant in the vicinity of the QCP can be used to extract the correlation-length exponent defined in Eq. (17). As illustrated in Fig. 4, we obtain an excellent collapse of data taken at different temperatures with a fitted exponent $v(r=0.4, s=0.6)^{-1}=0.25(3)$.

Having found $g_{c}$, we are able to establish that the static local susceptibility has the expected temperature dependence in each phase and at the critical coupling, as illustrated in Fig. 5. In particular, for $g=g_{c}, \chi_{\text {stat }}$ follows Eq. (16) with $x(r, s)=0.61(2)$ over the lowest decade of temperature for which data were obtained: $2.5 \times 10^{-4} D \leqslant T \lesssim T_{u} \simeq 2.5 \times$ $10^{-3} D$. (For comparison purposes, we note that for $g=0$, the Kondo temperature is $T_{K}^{0}=0.06 D$.) To within numerical accuracy, we find that $x(r, s)=x_{B}(s)=s$, in agreement with previous perturbative and numerical RG studies. ${ }^{21-23}$

In the BFK model, the convergence of the NRG manybody spectrum to the critical spectrum is fastest (i.e., the crossover scale $T_{u}$ is highest) for $g_{u} / D=0.84(4)$, with $J_{c}(g=$ $0.84 D) / 2 D \simeq 0.9666$. (The value of $J_{c}$ was determined to roughly 10 significant figures.)

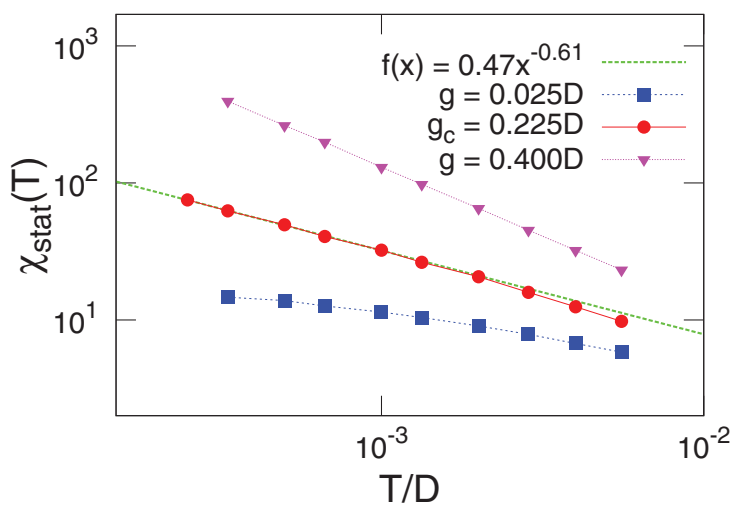

FIG. 5. (Color online) Static spin susceptibility $\chi_{\text {stat }}$ from CTQMC vs temperature $T$ for $r=0.4$ and $s=0.6$ in the Kondoscreened phase (squares), at the critical coupling (circles), and in the localized phase (triangles). At the critical coupling $g_{c} \approx 0.225$, $\chi_{\text {stat }}$ diverges according to Eq. (16) with $x=0.61(2)$. 


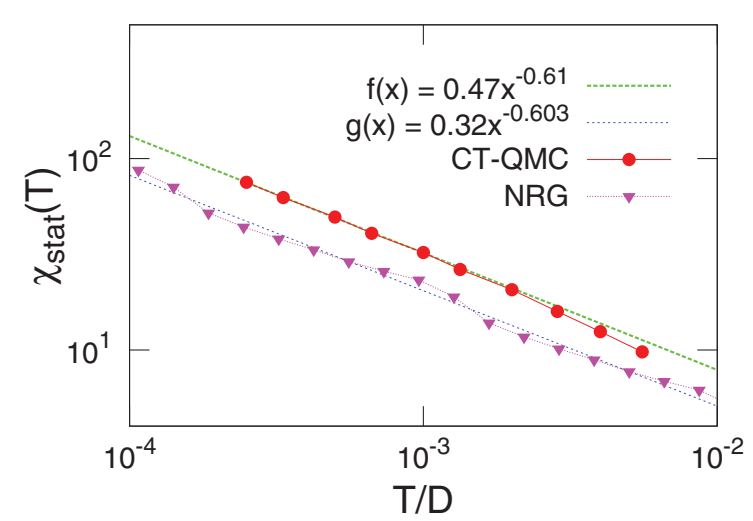

FIG. 6. (Color online) Static spin susceptibility $\chi_{\text {stat }}$ vs temperature $T$ for $r=0.4$ and $s=0.6$ calculated within CT-QMC for the BFA model and using the NRG for the BFK model at the QCP. The curves run parallel over the temperature range shown and are fitted by consistent exponents.

Figure 6 superimposes the temperature dependence of the critical static local susceptibilities of the Anderson and Kondo models. The NRG results for the Kondo model exhibit small oscillations around the dependence predicted in Eq. (16). Such oscillations, which are periodic in $\ln T$ with period $\ln \Lambda$, are a known consequence of the NRG band discretization ${ }^{50}$ that can be reduced in amplitude by working with smaller values of $\Lambda$. Over the two decades of temperature shown in the figure, the $\mathrm{NRG}$ data are described by an exponent $x \simeq 0.603$. However, a fit over the range $10^{-15} \leqslant T / D \leqslant 10^{-5}$ yields an improved estimate $x=0.600(1)$, consistent to within numerical error with the CT-QMC result for the Anderson model. Like the CTQMC estimate for the Anderson model, this value is consistent with the hypothesis that for $x_{B}(s)<x_{F}(r)$ spin fluctuations are primarily driven critical by the bosonic bath, and $x(r, s)=$ $x_{B}(s)=s$.

The reciprocal of the correlation-length exponent extracted from the crossover in the NRG many-body spectrum is $v^{-1}=0.233(1)$, again consistent with the value obtained for the Anderson model. This value differs from that found in two other cases: in the metallic BFK model, ${ }^{14} v(r=0, s=$ $0.6)^{-1}=0.509(1)$, while in the pseudogap Kondo model, ${ }^{18}$ $v_{F}(r=0.4)^{-1}=0.171(1)$. Although the critical spin fluctuations (and hence the exponent $x$ ) for $(r, s)=(0.4,0.6)$ seem to be dominated by the bosonic bath, the RG flow away from the critical point described by the exponent $v$ is clearly different from that in cases of pure-bosonic or pure-fermionic criticality.

\section{Results for $r=0.4, s=0.8$}

Figure 7 shows the variation of the BFA-model Binder cumulant for $r=0.4, s=0.8$ as calculated using CT-QMC. The intersection of curves representing different temperatures places the QCP at $g_{c} / D=0.28(1)$. This critical value is larger than that $\left(g_{c} / D \simeq 0.225\right)$ for $s=0.6$ because the bosonic interaction between time segments falls off faster with increasing $s$. Figure 8 shows that the Binder cumulant scales according to Eq. (12) with an excellent collapse of data taken at different temperatures with a fitted exponent $v(r=0.4, s=0.8)^{-1}=0.17(2)$.

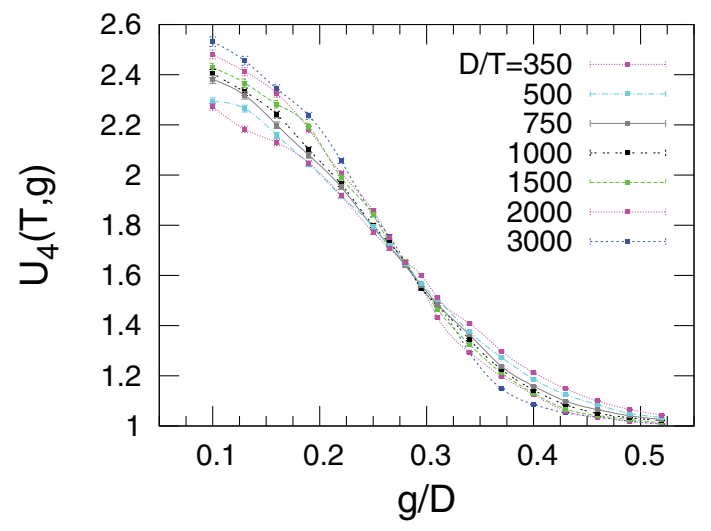

FIG. 7. (Color online) Binder cumulant $U_{4}$ vs bosonic coupling $g$ for $r=0.4$ and $s=0.8$ at different inverse temperatures $\beta=1 / T$. There is a clear intersection of the curves at the critical coupling $g_{c} / D=0.28(1)$.

Figure 9 illustrates the temperature dependence of the static local susceptibility in each phase and at the critical coupling. For $g=g_{c}, \chi_{\text {stat }}$ follows Eq. (16) with $x(r, s)=0.68(2)$ over the lowest decade of temperature for which data were obtained, a clear departure from the behavior $x(r, s)=x_{B}(s)=s$ seen above for $(r, s)=(0.4,0.6)$.

In the BFK model, the convergence of the NRG many-body spectrum to the critical spectrum is fastest (i.e., the crossover scale $T_{u}$ is highest) for $g_{u}=0$, with $J_{u} \equiv J_{c}\left(g_{u}\right) / 2 D \simeq$ 0.7908 . The value $g_{u}=0$ means that the impurity is entirely decoupled from the bosons, and the QCP must be of pure-fermionic character. Indeed, the asymptotic low-energy many-body spectrum in the quantum-critical regime can be reproduced by taking every possible combination of (i) one state from the spectrum of free $s=0.8$ bosons, and (ii) one state from the critical spectrum of the $r=0.4$ pseudogap Kondo model. We summarize this spectral decomposition in the shorthand $(\mathrm{BF}$ critical $)=(\mathrm{B}$ free $) \otimes(\mathrm{F}$ critical $)$ and refer to it in the following simply as F-type criticality.

Figure 10 compares the temperature dependence of the critical static local susceptibilities of the BFA and BFK models. Over the two decades of temperature shown in the figure, the NRG data for the BFK model are described by an exponent

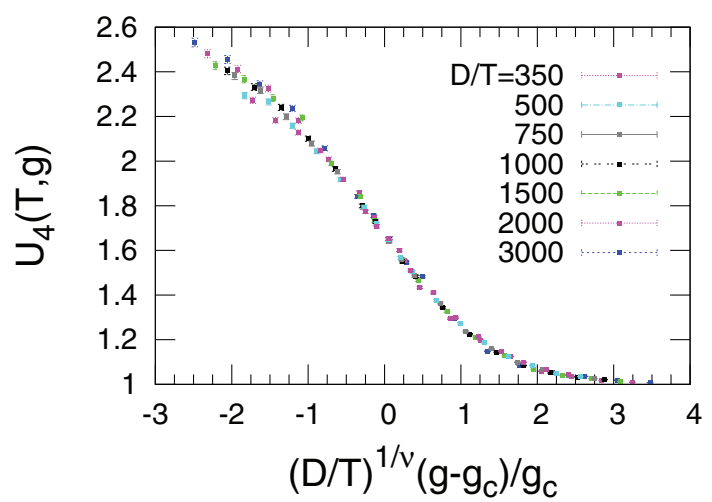

FIG. 8. (Color online) Data collapse of the Binder cumulant for $r=0.4$ and $s=0.8$. A correlation length exponent $v(r=0.4, s=$ $0.8)^{-1}=0.17(2)$ produces an excellent collapse in the vicinity of the critical point. 


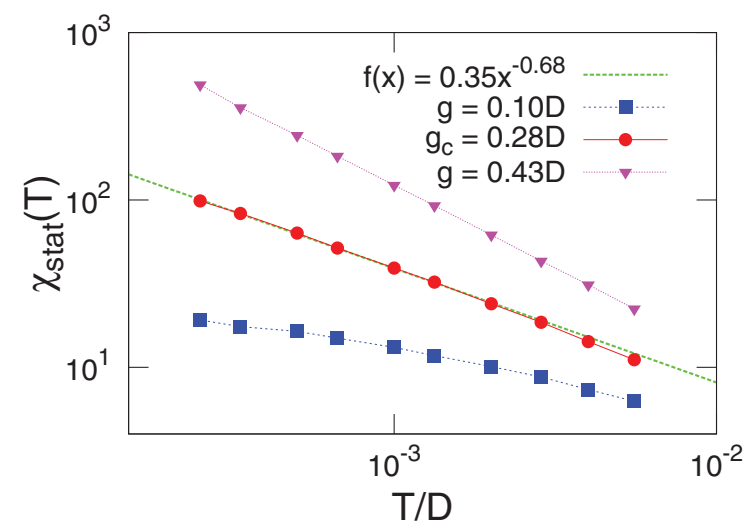

FIG. 9. (Color online) Static spin susceptibility $\chi_{\text {stat }}$ from CTQMC vs temperature $T$ for $r=0.4$ and $s=0.8$ in the Kondoscreened phase (squares), at the critical coupling (circles), and in the localized phase (triangles). At the critical coupling $g_{c} / D=0.28(1)$, $\chi_{\text {stat }}$ diverges according to Eq. (16) with $x=0.68(2)$.

$x \simeq 0.68$, identical to the CT-QMC value for the Anderson model. However, since the QCP corresponds to $g=0$, we know that the exponent must coincide exactly with that of the pseudogap Kondo model ${ }^{18}: x_{F}(r=0.4)=0.688(1)$. This value clearly differs from the one $x=s=0.8$ found within the spin-boson model ${ }^{41}$ and the metallic BFK model. ${ }^{13}$

Similarly, we can be confident that the correlation-length exponent must be identical to that of the pseudogap Kondo model $v_{F}(r=0.4)^{-1}=0.171(1)$, a result that is consistent with the CT-QMC value $v(r=0.4, s=0.8)^{-1}=0.17(2)$ quoted above.

The preceding results suggest that in cases $(r, s)$ where $x_{B}(s)>x_{F}(r)$, the physics at and near the QCP is determined primarily by fermionic fluctuations, and that critical properties should coincide with those of the pseudogap Anderson and pseudogap Kondo models.

\section{Results for other $(r, s)$}

In order to investigate more systematically the different types of quantum criticality exemplified in the cases

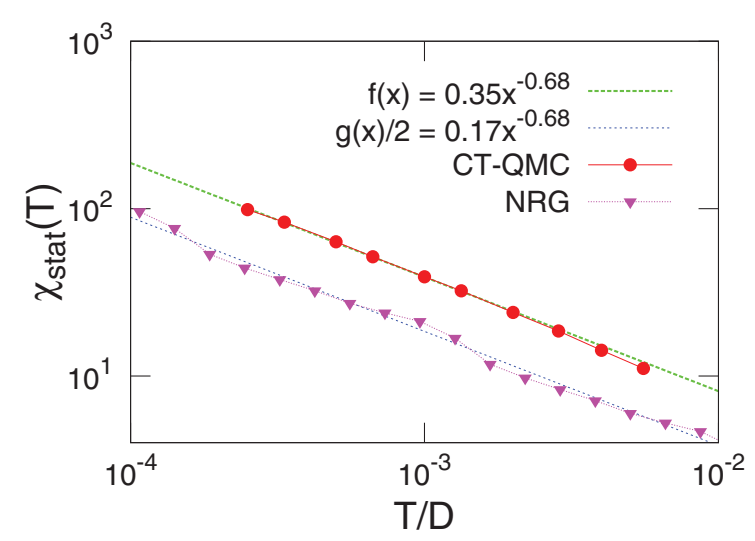

FIG. 10. (Color online) Static spin susceptibility $\chi_{\text {stat }}$ vs temperature $T$ for $r=0.4$ and $s=0.8$ calculated within CT-QMC for the BFA model and using the NRG for the BFK model at the QCP. The curves run parallel over the temperature range shown and are described by consistent exponents. $(r, s)=(0.4,0.6)$ and $(0.4,0.8)$, we have studied the particlehole-symmetric pseudogap BFK model for 23 different $(r, s)$ pairs spanning the ranges $0.1 \leqslant r \leqslant 0.4$ and $0.5 \leqslant s \leqslant 0.9$. Table I summarizes the critical properties, one line per $(r, s)$ pair. On each line, an estimate of $g_{u}$, the value of $g$ that produces the highest temperature $T_{u}$ of entry into the quantum-critical regime for $J \simeq J_{u} \equiv J_{c}\left(g_{u}\right)$, is followed by the assignment of the critical many-body spectrum to one of three categories $(\mathrm{F}, \mathrm{B}$, or $\mathrm{M})$ described further in the following.

The remaining columns of Table I list critical exponents: $x$, $\beta$, and $1 / \nu$ [defined in Eqs. (16), (19), and (17), respectively] are values calculated or inferred for the pair $(r, s)$ in question. For purposes of comparison, we also list $1 / v_{B}(s)$, the reciprocal of the order-parameter exponent at a pure-bosonic QCP with the same $s$, as determined within the metallic BFK model. The exponent $x$ has been calculated for all but a small number of $(r, s)$ pairs, and in all cases its value is consistent with Eq. (18). The order-parameter exponent $\beta$ can generally be evaluated to higher accuracy than $v$ (because the latter depends on $T_{l}$ values obtained by interpolation of data collected at a discrete set of temperature points), and has also been calculated for almost all $(r, s)$ pairs. By contrast, we have explicitly computed $1 / v$ for only about half the pairs. In every case where it can be tested, spanning the full range of bath exponents $0 \leqslant r<\frac{1}{2}$ and $\frac{1}{2} \leqslant s<1$, hyperscaling [Eq. (22)] holds to within our estimated numerical uncertainty. For this reason, we can confidently apply Eq. (22) to predict the value of $1 / v$ in cases where it has not been computed directly. Finally, we note that wherever it has been calculated, $\delta$ [defined in Eq. (20), but not listed in Table I] obeys Eq. (23) to high precision.

Based on an examination of the critical spectra and the exponents $x$ and $\beta$ (and hence $v$ via hyperscaling), we are able to identify three distinct types of quantum criticality:

(i) Fermionic (F). The asymptotic low-energy critical spectrum exhibits $S U(2)$ spin symmetry and decomposes into a direct product of the spectrum of free bosons with bath exponent $s$ and the critical spectrum of the pseudogap Kondo model with band exponent $r$, i.e., $(\mathrm{BF}$ critical $)=(\mathrm{B}$ free) $\otimes(\mathrm{F}$ critical). All static critical exponents that have been calculated are identical to those of the pure-fermionic pseudogap Anderson and Kondo models with the same $r$.

(ii) Bosonic (B): The asymptotic low-energy critical spectrum exhibits $\mathrm{SU}(2)$ spin symmetry and decomposes into a direct product of the critical spectrum of the spin-boson model with bath exponent $s$ and the strong-coupling spectrum ${ }^{51}$ of the pseudogap Kondo model with band exponent $r$, i.e., (BF critical $)=($ B critical $) \otimes($ F strong coupling $)$. All static critical exponents that have been calculated in region $\mathrm{B}$ are identical to those of the spin-boson model and of the metallic $(r=0)$ BFA and BFK models with the same $s$. However, despite the asymptotic decomposition of the low-energy spectrum, the single-particle spectral function has a non-Fermi liquid form (at least for $r=0$ ), as discussed in Sec. V B. This may be related to the fact that the fermionic strong-coupling spectrum is not only found at the Kondo fixed point where the exchange couplings take renormalized values $J_{z}=J_{\perp}=\infty$, but also is approached for $J_{z}=\infty, J_{\perp}=$ finite in the limit of energy scales much smaller than $J_{\perp}$. We will return to this observation in Sec. V C. 
TABLE I. Summary of critical properties of the Bose-Fermi Kondo (BFK) model with pseudogap exponent $r$ and bosonic bath exponent $s$. As defined in the text, $g_{u}$ is the value of the bosonic coupling $g$ that yields the highest temperature of entry into the quantum-critical regime. In the fourth column, "F" means that the asymptotic low-energy form of the critical spectrum is a direct product of (i) the spectrum of free bosons with the same $s$ and (ii) the critical spectrum of the pseudogap Kondo model with the same $r$; "B" indicates that the asymptotic low-energy NRG critical spectrum is a direct product of (i) the critical spectrum of the spin-boson model with the same value of $s$ and (ii) the Kondo spectrum of the pseudogap Kondo model with the same $r$; and "M" means that the critical spectrum does not decompose into a direct product of bosonic and fermionic parts. Exponents $x, \beta$, and $v$ are as defined in Eqs. (16), (19), and (17), respectively, while $1 / v_{B}(s)$ is the reciprocal of the order-parameter exponent at a pure-bosonic QCP with the same $s$, as calculated in the metallic $(r=0) \mathrm{BFK}$ model. All values of $1 / v_{B}$ and those values of $1 / v$ followed by an asterisk were obtained from the corresponding value of $\beta$ using hyperscaling [Eq. (22)] under the assumption that $x=s$. Any exponent followed by " $§$ " has been set to that for the pure-fermionic pseudogap Kondo model (see the line labeled "no bosons" for each value of $r$ ) since $g_{u}=0$ indicates that the bosonic bath plays no part in the criticality. A number in parentheses represents the uncertainty in the last digit, equal to 1 where omitted.

\begin{tabular}{|c|c|c|c|c|c|c|c|}
\hline$r$ & $s$ & $g_{u} / D$ & Critical spectrum & $x$ & $\beta$ & $1 / v$ & $1 / v_{B}(s)$ \\
\hline \multirow[t]{7}{*}{0.1} & 0.5 & $>10$ & B & 0.499 & & 0.48 & 0.475 \\
\hline & 0.7 & $6-8$ & B & 0.700 & 0.296 & $0.506^{*}$ & 0.506 \\
\hline & 0.75 & $6-8$ & B & & 0.254 & $0.493^{*}$ & 0.493 \\
\hline & 0.8 & $5.0(5)$ & B & 0.800 & 0.213 & 0.46 & 0.470 \\
\hline & 0.85 & $3.58(3)$ & M & & 0.176 & $0.426^{*}$ & 0.433 \\
\hline & 0.9 & $2.8(3)$ & M & 0.900 & 0.142 & 0.352 & 0.376 \\
\hline & No bosons & & & 0.989 & 0.060 & $0.093^{*}$ & \\
\hline \multirow[t]{8}{*}{0.2} & 0.5 & $>10$ & B & 0.499 & 0.526 & 0.48 & 0.475 \\
\hline & 0.6 & $5-10$ & B & & 0.394 & $0.508^{*}$ & 0.509 \\
\hline & 0.65 & $3.40(5)$ & M & & 0.347 & $0.504^{*}$ & 0.512 \\
\hline & 0.7 & $2.98(3)$ & M & & 0.313 & $0.479^{*}$ & 0.506 \\
\hline & 0.75 & $2.63(3)$ & M & & 0.282 & $0.443^{*}$ & 0.493 \\
\hline & 0.8 & $2.30(5)$ & M & 0.800 & 0.251 & 0.398 & 0.470 \\
\hline & 0.9 & 1.4 & M & 0.900 & 0.189 & $0.265^{*}$ & 0.376 \\
\hline & No bosons & & & 0.948 & 0.160 & $0.161^{*}$ & \\
\hline \multirow[t]{6}{*}{0.3} & 0.5 & 2.5 & M & 0.500 & 0.582 & $0.427(4)$ & 0.475 \\
\hline & 0.6 & $2.05(5)$ & M & 0.600 & 0.493 & $0.405(2)$ & 0.509 \\
\hline & 0.7 & $1.65(5)$ & M & 0.700 & 0.422 & $0.356^{*}$ & 0.506 \\
\hline & 0.8 & 1.1 & M & 0.800 & 0.365 & 0.270 & 0.470 \\
\hline & 0.9 & 0 & $\mathrm{~F}$ & $0.862 \S$ & $0.355 \S$ & $0.194 \S$ & 0.376 \\
\hline & No bosons & & & 0.862 & 0.355 & $0.194^{*}$ & \\
\hline \multirow[t]{6}{*}{0.4} & 0.5 & $1.15(5)$ & M & 0.500 & 0.930 & 0.269 & 0.475 \\
\hline & 0.6 & $0.84(2)$ & M & 0.600 & 0.853 & 0.233 & 0.509 \\
\hline & 0.7 & 0 & $\mathrm{~F}$ & $0.688 \S$ & $0.914 \S$ & $0.171^{*} \S$ & 0.506 \\
\hline & 0.8 & 0 & $\mathrm{~F}$ & $0.688 \S$ & $0.914 \S$ & $0.171^{*} \S$ & 0.470 \\
\hline & 0.9 & 0 & $\mathrm{~F}$ & $0.688 \S$ & $0.914 \S$ & $0.171^{*} \S$ & 0.376 \\
\hline & No bosons & & & 0.688 & 0.914 & $0.171^{*}$ & \\
\hline
\end{tabular}

(iii) Mixed (M): The critical spectrum exhibits broken SU(2) spin symmetry and does not decompose into a direct product of bosonic and fermionic parts. The exponents satisfy $x=x_{B}(s)=s$, but the order-parameter exponent lies between the values for the spin-boson model and the pseudogap Kondo/Anderson models, i.e., $v_{F}^{-1}(r)<v^{-1}(r, s)<v_{B}^{-1}(s)$.

The three types of quantum criticality are clearly revealed in plots of $x$ and $1 / v$ versus $s$ at fixed $r$. As can be seen in Fig. 11, $x(r, s)$ coincides with $x_{B}(s)=s$ until the latter value exceeds its counterpart $x_{F}(r)$ in the pure-fermionic pseudogap problem. The break in the slope of $x$ versus $s$ marks the transition from M-type to F-type criticality. Figure 12 shows that the Bose-Fermi correlation-length exponent equals the bosonic value $v_{B}(s)$ for sufficiently small $s$ (B-type criticality) and equals the fermionic value $v_{F}(r)$ over precisely the range of $s$ where $x(r, s)=x_{F}(r)$ (F-type criticality), but between these regimes, $v(r, s)$ takes values that differ from both $v_{B}(s)$ and $v_{F}(r)$ (M-type criticality).

Figure 13 summarizes the type of criticality found at different locations on the $r-s$ plane, including points studied for the metallic case $r=0$ where the behavior is always of the B type. It is seen that each type of criticality (F, B, or M) occupies a contiguous region. All the results are consistent with there being a boundary $s=x_{F}(r)$ between the $\mathrm{F}$ and $\mathrm{M}$ regions (shown as a solid curve in Fig. 13). As argued at the beginning of this section, such a boundary arises from the assumption that the spin response at the Bose-Fermi critical point is dominated by the bath (bosonic or fermionic) that has the more singular dynamical spin fluctuations, corresponding to the smaller value of $x$. The results in Fig. 13 are also consistent with there being a boundary $s=1-2 r$ between the $\mathrm{M}$ and $\mathrm{F}$ regions (the straight line in the figure). Such a 


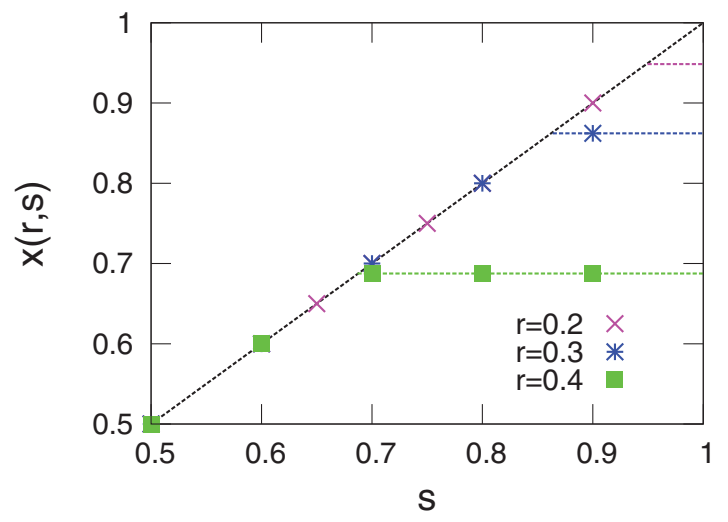

FIG. 11. (Color online) Static magnetic critical exponent $x$ vs bath exponent $s$ for $r=0.2,0.3$, and 0.4. The diagonal line represents the pure-bosonic exponent $x_{B}(s)=s$, while each horizontal line segment shows the pure-fermionic value $x_{F}(r)$. For each $(r, s)$ pair, the Bose-Fermi exponent satisfies $x=\min \left[x_{F}(r), x_{B}(s)\right]$.

boundary marks the line of equality of the frequency exponents of the bare bosonic propagator and the fermionic particle-hole bubble, ${ }^{52}$ although the significance of this observation in the present context remains to be established.

\section{B. Critical dynamics}

We now turn to the finite-temperature dynamics of the impurity Green's function and the local susceptibility calculated at the critical bosonic coupling $g=g_{c}$. Since the NRG is unreliable in the regime $|\omega| \lesssim T$, we rely mainly on the CT-QMC for this part of the study, focusing once more on the type-M case $r=0.4, s=0.6$ and on the type-F case $r=0.4$, $s=0.8$.

Figures 14 and 15 plot $G(\tau, T)$ and $\chi_{\mathrm{loc}}(\tau, T)$, respectively, as functions of the combination $\xi=\pi \tau_{0} T / \sin (\pi \tau T)$, where $\tau_{0}=1 / D$ renders the scaling function dimensionless. For both

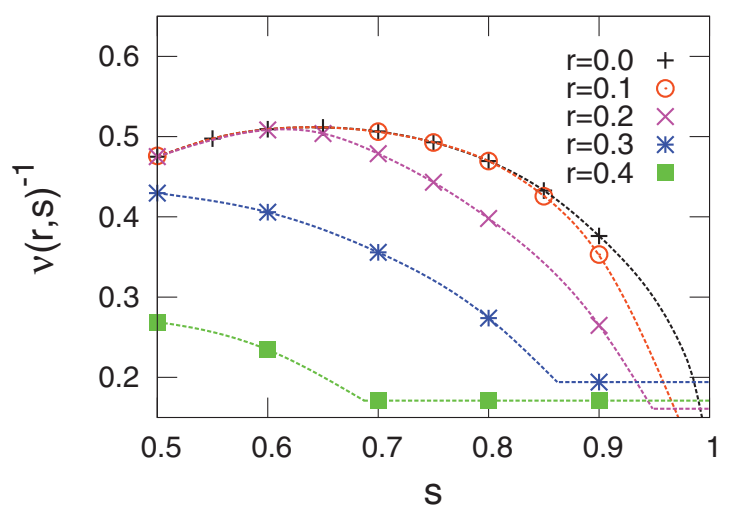

FIG. 12. (Color online) Reciprocal of the correlation-length exponent $1 / v$ vs bath exponent $s$ for the metallic case $r=0$ and for pseudogaps described by $r=0.1-0.4$. The $r=0$ exponents coincide with those of the corresponding spin-boson model and represent the values $1 / v_{B}(s)$ describing pure-bosonic criticality. Each horizontal line segment shows a pure-fermionic value $1 / v_{F}(r)$. For each $(r, s)$, the Bose-Fermi exponent coincides with $1 / v_{B}(s)$ for $s<1-2 r$ and with $1 / v_{F}(r)$ for $s \geqslant x_{F}(r)$. For $1-2 r<s<x_{F}(r), 1 / v(r, s)$ lies in-between the bosonic and fermionic values.

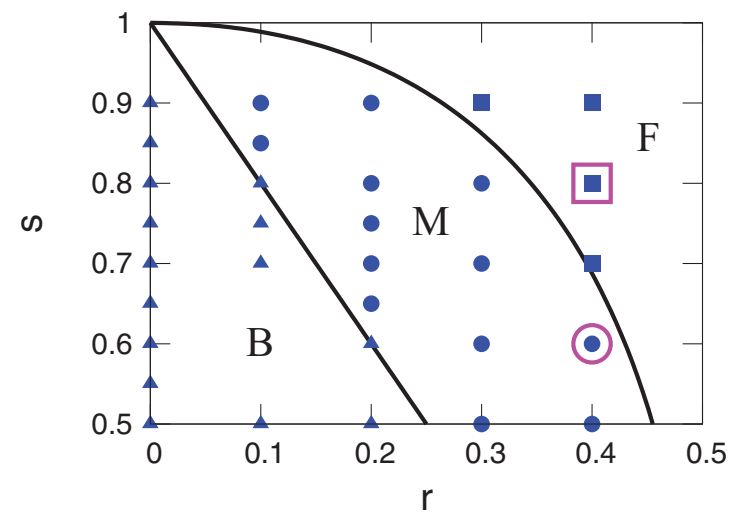

FIG. 13. (Color online) Summary of the pairs of bath exponents $(r, s)$ studied in this work. Results for $r=0$ describe a metallic conduction band, while those for $r>0$ correspond to pseudogapped problems. Squares, triangles, and circles, respectively, correspond to quantum criticality of the F, B, and M types, as described in the text. Filled symbols summarize NRG results for the BFK model, while open symbols represent CT-QMC results for the BFA model. Solid lines show the conjectured boundaries $s=1-2 r$ and $x_{B}(s)=s=x_{F}(r)$ between the different types of criticality.

$(r, s)$ pairs, the critical correlation functions at temperatures well below the bare Kondo temperature $T_{K}^{0} \equiv T_{K}(g=0) \approx$ $0.06 D$ exhibit excellent scaling collapse over two decades of $\xi$. The scaling collapse leads to the important conclusions that in the long-time limit $\tau T_{K}^{0} \gg 1$,

$$
\begin{gathered}
G(\tau, T)=\Psi\left(\frac{\pi \tau_{0} T}{\sin (\pi \tau T)}\right) \stackrel{T \ll T_{K}^{0}}{\sim}\left(\frac{\pi \tau_{0} T}{\sin (\pi \tau T)}\right)^{\eta_{G}}, \\
\chi_{\mathrm{loc}}(\tau, T)=\Phi\left(\frac{\pi \tau_{0} T}{\sin (\pi \tau T)}\right) \stackrel{T \ll T_{K}^{0}}{\sim}\left(\frac{\pi \tau_{0} T}{\sin (\pi \tau T)}\right)^{\eta_{\chi}} .
\end{gathered}
$$

We obtain $\eta_{G}=0.58(4), \eta_{\chi}=0.40(2)$ for $s=0.6$ and $\eta_{G}=$ $0.58(4), \eta_{\chi}=0.31(2)$ for $s=0.8$. These exponents are consistent with the relations

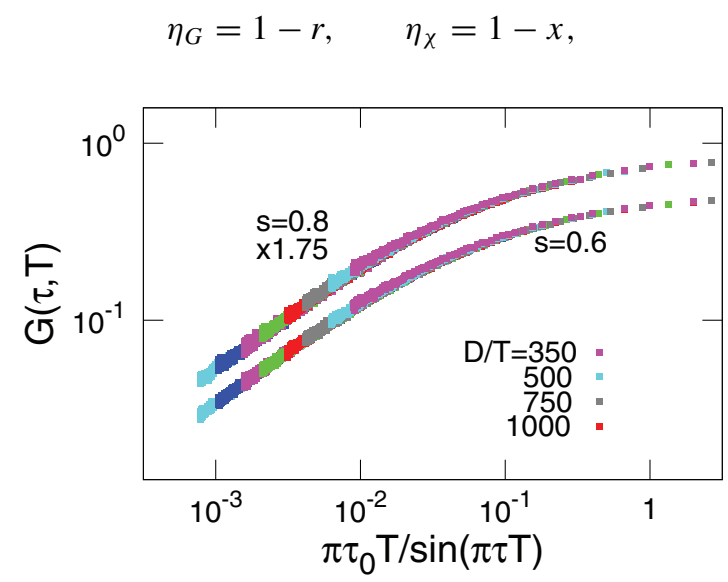

FIG. 14. (Color online) Single-particle Green's function $G(\tau, T)$ vs $\xi=\pi \tau_{0} T / \sin (\pi \tau T)$ for $r=0.4, s=0.6, g=0.225 D \approx g_{c}$ (lower data) and for $r=0.4, s=0.8, g=0.28 D \approx g_{c}$ (upper data, all $G$ values multiplied by 1.75 to avoid overlap with the $s=0.6$ data). One observes excellent collapse of the data for just under two decades of $\xi$. Temperature labels are shared between this figure and Fig. 15. 


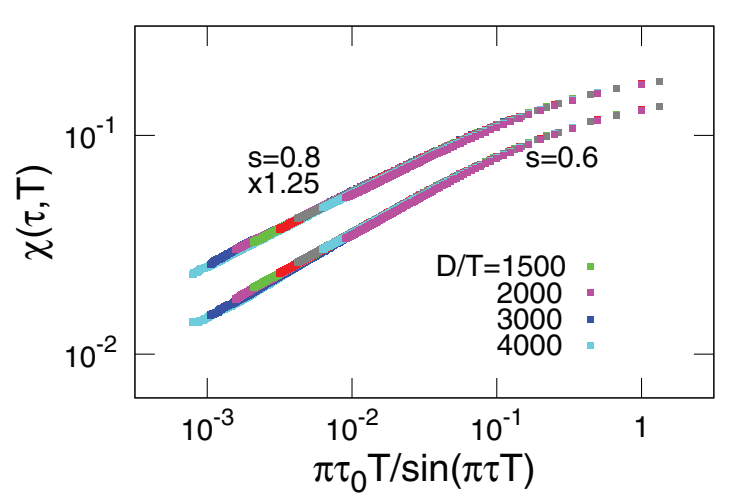

FIG. 15. (Color online) Local spin susceptibility $\chi(\tau, T)$ vs $\xi=$ $\pi \tau_{0} T / \sin (\pi \tau T)$ for $r=0.4, s=0.6, g=0.225 D \approx g_{c}$ (lower data) and for $r=0.4, s=0.8, g=0.28 D \approx g_{c}$ (upper data, all $\chi$ values multiplied by 1.25 to avoid overlap with the $s=0.6$ data). One observes excellent collapse of the data for over two decades of $\xi$. Temperature labels are shared between this figure and Fig. 14.

where $x(r, s)$ is defined in Eq. (16). In the zero-temperature limit, Eq. (24) and the first Eq. (25) give $G(\tau, T \rightarrow 0) \sim$ $\tau^{-(1-r)}$, reproducing an exact result. ${ }^{21,22}$ The quality of the scaling collapse in Figs. 14 and 15, as well as the reproduction of the correct zero-temperature limit, provide significant evidence that our results have reached the asymptotic lowenergy scaling regime.

Since $1-r$ and $1-x$ are both less than one, each correlator obeys $\omega / T$ scaling per the discussion of Eq. (1). The observation that $\eta_{\chi} \neq 2 \eta_{G}$ implies that vertex corrections can not be neglected, in line with the fully interacting nature of the QCP.

For reasons discussed in Sec. VI, we have not been able to access low enough temperatures using CT-QMC to study cases of B-type quantum criticality. In this region of the $r-s$ plane, we must rely on information from previous NRG studies of the $r=0$ BFK and BFA models, ${ }^{13,14}$ which have demonstrated that at the critical coupling, the local susceptibility calculated for $|\omega| \gtrsim T$ is consistent with the existence of $\omega / T$ scaling, while the zero-temperature impurity spectral function shows a non-Fermi-liquid form. The latter is also seen for $r=0$ results in a dynamical large- $N$ limit, ${ }^{53}$ as a function of both frequency and temperature. It is reasonable to attribute this non-Fermi-liquid behavior to the existence of a RG-irrelevant coupling between the fermionic and bosonic sectors of the Hilbert space.

\section{RG flow diagrams}

We conclude this section with a discussion of the RG structure of the Ising-anisotropic pseudogap BFK model in the different regions of the $r-s$ plane. It is important to recognize that the bare couplings $(g, J)$ entering Eq. (3) are insufficient to describe this RG structure. Since a coupling $g>0$ breaks spin-rotation symmetry, $\mathrm{SU}(2)$ symmetry of the Kondo exchange need not be preserved under renormalization, and a minimal description involves keeping track of a triad of effective couplings $\left(g, J_{z}, J_{\perp}\right)$.

The stable fixed points of the pseudogap BFK model are the Kondo fixed point at $g_{K}=0, J_{z, K}=J_{\perp, K}=\infty$ and a line of localized fixed points at $g_{L}=\infty, J_{\perp, L}=0$, and (at least for $r=0$; see Ref. 14) $J_{z, L} \propto\left(g-g_{c}\right)^{-\beta}$ where $g$ is the bare bosonic coupling and $\beta$ is the order-parameter exponent. The QCP of the pseudogap Kondo model is located at $g_{F}=0$ and $J_{z, F}=J_{\perp, F}=J_{c}(g=0)$, where $\rho_{0} J_{c} \rightarrow r$ for $r \rightarrow 0$.

A perturbative RG treatment of the model valid for $r=0$ and $0<1-s \ll 1$ has shown ${ }^{11}$ that the Bose-Fermi QCP is located at $K_{0} g^{*}=O(1), J_{z}^{*}=\infty$, and $\rho_{0} J_{\perp}^{*} \simeq \sqrt{1-s} \ll 1$, with the NRG having confirmed the result $J_{z}^{*}=\infty$ over a wider range of $s$ values. ${ }^{14}$ What remains to be established is the manner in which $\left(g^{*}, J_{z}^{*}, J_{\perp}^{*}\right)$ evolves with increasing $r$ and/or $s$ to reach $\left[0, J_{c}(0), J_{c}(0)\right]$ upon entry into the region $\mathrm{F}$ of the $r$-s plane. Since region $\mathrm{F}$ is bounded by the line $x_{F}(r)=s$, and the exponent $x_{F}(r)$ is very well described ${ }^{18}$ by $x_{F}=$ $1-\left[\rho_{0} J_{c}(0)\right]^{2}=1-\left[\rho_{0} J_{\perp}^{*}\right]^{2}$, one is led to conclude that for a given value of $s$ satisfying $0<1-s \ll 1$, the relation $\rho_{0} J_{\perp}^{*} \simeq$ $\sqrt{1-s}$ holds true both at $r=0$ (on the leftmost edge of region $\mathrm{B}$ in Fig. 13) and at the border between regions $\mathrm{M}$ and $\mathrm{F}$. This observation, when combined with the asymptotic spectral decomposition $(\mathrm{BF}$ critical $)=(\mathrm{B}$ critical $) \otimes(\mathrm{F}$ strong coupling $)$ that holds throughout region $\mathrm{B}$, naturally leads to the conjecture that upon increasing $r$ from 0 to the point of entry into region F, (1) $J_{\perp}^{*}$ remains constant (or very nearly so); (2) $K_{0} g^{*}$ decreases monotonically from a value of order unity to reach zero; and (3) $J_{z}^{*}$ is infinite throughout region $\mathrm{B}$, and decreases monotonically to $J_{c}(r)$ on crossing region $\mathrm{M}$. The presence in region $\mathrm{M}$ of finite exchange couplings satisfying $J_{z}^{*}>J_{\perp}^{*}$ is consistent with the observation of broken $\mathrm{SU}(2)$ spin symmetry in the asymptotic low-energy critical spectrum. By contrast, the value $J_{z}^{*}=\infty$ in region B ensures that the spectrum appears to be $S U(2)$ invariant at energy scales much below $J_{\perp}^{*}$.

Support for this picture comes from Table I, which shows a clear trend with increasing $r$ at fixed $s$ (or with increasing $s$ at fixed $r$ ) in the value $g_{u}$ of the bosonic coupling that brings the model into its quantum-critical regime at the highest temperature. Throughout region B in Fig. 13, $g_{u}$ and the corresponding Kondo coupling $J_{u}=J_{c}\left(g_{u}\right)$ are very large in order to achieve rapid flow to $J_{z}^{*}=\infty$ and the large fixed-point value of $K_{0} g^{*}$. While region M is crossed, $g_{u}$ and $J_{u}$ decrease in line with $g^{*}$ and $J_{z}^{*}$. In region $\mathrm{F}$, the value $g_{u}=0$ shows that the critical point can be reached without any coupling of the impurity to the bosons, meaning that $g^{*}$ is necessarily zero and that $J_{z}^{*}=J_{\perp}^{*}=J_{u}$.

Based on the preceding considerations, we propose the schematic RG flow diagrams shown projected onto the $g-J_{\perp}$ plane in Fig. 16. In regions B and M, a QCP labeled BFC in Fig. 16(a) lies on the separatrix between the basins of attraction of the Kondo and localized fixed points ( $\mathrm{K}$ and $\mathrm{L}$, respectively). RG flow along the separatrix is toward BFC, and on the small- $g$ side, away from the fermionic pseudogap critical point FC. With increasing $r$ at fixed $s$ (or increasing $s$ at fixed $r$ ), BFC moves to smaller values of $g$, and merges with $\mathrm{FC}$ at the boundary between regions $\mathrm{M}$ and F. Throughout the latter region of the $r$-s plane, the RG structure is as shown in Fig. 16(b), with flow along the separatrix toward FC.

\section{Effect of particle-hole asymmetry}

To this point, we have focused exclusively on conditions of strict particle-hole $(p-h)$ symmetry, i.e., $U=-2 \epsilon_{d}$ for 


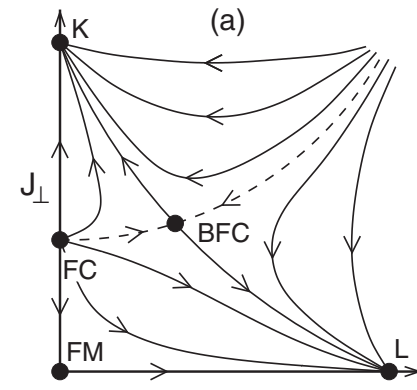

g

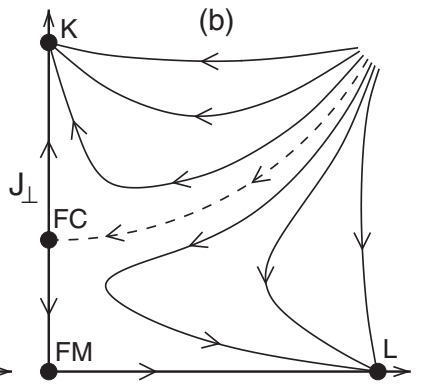

g
FIG. 16. Schematic RG flow diagrams for the pseudogap BFK model projected onto the plane spanned by the impurity-boson coupling $g$ and the spin-flip exchange scattering $J_{\perp}$, valid for bath exponents $(r, s)$ such that the quantum criticality is of the (a) B or M, and (b) F types. Not shown in these diagrams is a third axis describing the longitudinal exchange coupling $J_{z}$. The dashed line marks the boundary between the Kondo and localized phases. Arrows show the direction of RG flow of the effective couplings between fixed points represented by circles: free moment $\left(\mathrm{FM}\right.$, at $\left.J_{z}=0\right)$, Kondo $(\mathrm{K}$, at $J_{z}=\infty$ ), localized (L, a line of fixed points spanning $0 \leqslant J_{z} \leqslant \infty$ ), fermionic critical (FC, at $J_{z}=J_{\perp}$ ), and Bose-Fermi critical (BFC, at $J_{z}=\infty$ in region B but at finite $J_{z}>J_{\perp}$ in region M). Very similar RG flow diagrams can be constructed for the pseudogap BFA model.

the BFA model, which maps to a BFK model with potential scattering $W=0$. In this section, we consider the effects of breaking this symmetry, supporting our arguments with NRG results for the critical spectrum and for the critical exponent $x$ entering Eq. (16), as obtained in a preliminary study of the BFK model with $W \neq 0$.

Under the conditions of $p$ - $h$ symmetry defined in the previous paragraph, the pseudogap Kondo and Anderson models exhibit a Kondo-destruction QCP only over the range of band exponents $0<r<\frac{1}{2}$; for $r \geqslant \frac{1}{2}$, the Kondo phase disappears and over the entire parameter space the system approaches the FM fixed point at low temperatures. In these models, it is known that away from $p$ - $h$ symmetry, a strongcoupling or Kondo phase is present for all $r>0$, and that for $0<r<1$ this phase is separated from the free-moment phase by an interacting QCP. For exponents $0<r<r^{*} \approx 0.375$, $p$ - $h$ asymmetry is irrelevant at the QCP and the quantum criticality is identical to that at $p-h$ symmetry, whereas for $r^{*}<r<1$, quantum criticality away from $p$ - $h$ symmetry is governed by a distinct asymmetric QCP. ${ }^{17}$

Combining these well-established properties of the purefermionic pseudogap models with the results presented in Sec. V allows informed speculation about the effects of $p$ - $h$ asymmetry in the pseudogap BFA and BFK models. We expect the persistence of a region of B-type criticality in which the critical spectrum has the product form $(\mathrm{BF}$ critical $)=(\mathrm{B}$ critical $) \otimes($ F strong coupling), with $p$ - $h$ asymmetry affecting the fermionic strong-coupling spectrum but not the critical properties studied in this work, all of which are determined solely by the bosonic spectrum. For $r=0, p$ - $h$ asymmetry should be marginal (as it is in the metallic Anderson and Kondo models), giving rise to a line of QCPs sharing the same critical properties. For $r>0$, by contrast, $p$ - $h$ asymmetry should be relevant, with the fermionic spectrum being that of the asymmetric strong-coupling fixed point. These conjectures are consistent with preliminary NRG studies of the cases $(r, s)=(0,0.7)$ and $(0.1,0.7)$.

In the presence of $p$ - $h$ asymmetry, we also expect a region in which the dynamical spin response arising from the fermions is more singular than that from the bosonic bath. This region of what we will dub $\mathrm{F}^{\prime}$-type criticality is likely to span the range of exponents $0<r<1$ and $\frac{1}{2}<r<1$ in which $s>x_{F}^{\prime}(r)$. Here, $x_{F}^{\prime}(r)$ is the value of the exponent $x$ in Eq. (16) at the asymmetric pseudogap QCP. For $0<r<r^{*}, x_{F}^{\prime}=x_{F}$ (Ref. 18) so $\mathrm{F}^{\prime}$ criticality should be identical to $\mathrm{F}$ type. For $r^{*}<r<1$, by contrast, $x_{F}^{\prime}>x_{F}$ (Ref. 18), and the $\mathrm{F}^{\prime}$ critical exponents should belong to the universality class of the asymmetric pseudogap QCP. We have found an example of $\mathrm{F}^{\prime}$ criticality for $(r, s)=(0.4,0.9)$.

Finally, it seems probable that the $B$ and $F^{\prime}$ regions will be separated by one in which the critical spectrum does not have a simple direct product form. At $p$ - $h$ symmetry, the M-type region covers the range $1-2 r<s<x_{F}(r)$. As discussed in Sec. V A3, the lower bound on the range of $s$ seems to be defined by the equality of the frequency exponents of the bare bosonic propagator and the fermionic particle-hole bubble. Since $p-h$ asymmetry is irrelevant at the FM fixed point, ${ }^{17}$ there seems to be no reason to expect the boundary to be affected by this breaking of symmetry. On the other hand, the upper bound $s=x_{F}(r)$ seems likely to be replaced by $s=x_{F}^{\prime}(r)$, the condition discussed in the previous paragraph for entry into a region of $\mathrm{F}^{\prime}$ criticality.

\section{SUMMARY}

In this work, we have applied a combination of continuoustime quantum Monte Carlo (CT-QMC) and numerical renormalization-group (NRG) methods to study systematically the interplay between bosonic and fermionic baths, each of which on its own can induce critical Kondo destruction at a continuous zero-temperature transition. We have shown that at particle-hole symmetry, the quantum-critical point (QCP) in the easy-axis pseudogap BFA model belongs to the same universality class as the QCP of the corresponding Kondo model. We have further shown the surprising result that the value of the exponent $x$ for the temperature dependence of the critical local spin susceptibility [defined in Eq. (16)] of either model is sensitive to the exponents $r$ and $s$ characterizing the vanishing of the fermionic and bosonic densities of states. In the region of the $r-s$ plane where $s \geqslant x_{F}(r)\left(x_{F}\right.$ being the thermal critical exponent of the pseudogap Anderson and Kondo models without bosons), all critical exponents of the Bose-Fermi models that we have calculated are identical to those of the pure-fermionic models, and the critical many-body spectrum decomposes into a direct product of a free bosonic spectrum and a critical pseudogap fermionic spectrum; this regime has eluded all previous studies. For $s<x_{F}(r)$, the critical spin fluctuations are instead dominated by the bosonic bath, leading to $x=x_{B}(s)=s$. However, the correlationlength exponent $v$ [defined in Eq. (17)] coincides with that of the spin-boson model, and the asymptotic low-energy critical spectrum decomposes into a direct product of a critical bosonic spectrum and a pseudogap Kondo fermionic spectrum, only for $s \leqslant 1-2 r$. Within an intermediate region $1-2 r<s<x_{F}(r), v(r, s)$ takes a value lying between those 
for the spin-boson model and for the pseudogap Anderson and Kondo models, and bosonic and fermionic degrees of freedom can not be disentangled in the critical spectrum. In all three regions, other static critical exponents are related to $x$ and $v$ via hyperscaling relations that are expected to hold only at an interacting critical point.

We have also shown that at the QCP, the imaginary-time correlation functions $G(\tau, T)$ and $\chi_{\operatorname{loc}}(\tau, T)$ scale as functions of $\xi=\pi T \tau_{0} / \sin (\pi \tau T)$ and that their real-frequency counterparts obey $\omega / T$ scaling, consistent with the notion that the QCP is fully interacting. Scaling collapse of imaginary-time correlators as functions of $\xi$ has previously been reported for the sub-Ohmic BFK model, ${ }^{31}$ where the Kondo effect is critically destroyed by the bosonic bath, and in the pseudogap Anderson model at and away from particle-hole symmetry, ${ }^{20,32}$ where criticality is driven by fermionic fluctuations of the band. That it generalizes to the more complex case considered in this work suggests that the scaling collapse may very well be a general feature of local quantum criticality. The scaling collapse in terms of $\xi$ implies (under conditions spelled out in Sec. V B) that the associated real-frequency correlator displays $\omega / T$ scaling, a property that has been reported in several experiments on unconventional quantum criticality in $4 f$-electron-based magnets. ${ }^{4,5}$ A scaling collapse of the form observed here is natural for boundary-conformal quantum impurity systems. However, conformal symmetry is broken both by the bosonic bath (as in the sub-Ohmic BFK model) and the fermionic bath (as in the pseudogap Anderson model). Symmetry restoration frequently accompanies criticality. The case discussed here, however, has to be distinguished from this more standard situation of irrelevant symmetry-breaking fields, as the broken symmetry in the bulk induces boundary criticality with local correlators that are compatible with a boundary-conformal critical theory. As discussed earlier, ${ }^{31}$ a deeper understanding of this observation should help identify a critical field theory of unconventional quantum criticality.

From a methodological viewpoint, this work has shown that the CT-QMC method can attain sufficiently low temperatures in the presence of a bosonic bath to identify a quantum-critical point lying between stable phases, and to obtain critical properties in agreement with those given by the NRG. Such study is possible using the CT-QMC only in cases where entry into the critical power-law regime takes place at a fairly high temperature $T_{u}$. The presence of a pseudogap in the fermionic density of states helps in this regard: the value of $T_{u}$ decreases as $r$ decreases, so larger $r$ values are optimal. Decreasing the bath exponent $s$ also reduces $T_{u}$, making it difficult to study the range $s<\frac{1}{2}$ without fine tuning of the Hamiltonian.

\section{ACKNOWLEDGMENTS}

We thank M. T. Glossop, P. Ribeiro, M. Vojta, F. Zamani, and J.-X. Zhu for useful discussions. This work has been supported by NSF Grants No. DMR-0710540, No. DMR1309531, and No. DMR-1107814, the Robert A. Welch Foundation Grant No. C-1411, and the Alexander von Humboldt Foundation. We acknowledge the hospitality of the Max Planck Institute for the Physics of Complex Systems (K.I. and J.H.P.), the Aspen Center for Physics (NSF Grant No. 1066293; S.K. and Q.S.), the Institute of Physics of Chinese Academy of Sciences, and Karlsruhe Institute of Technology (Q.S.).

\footnotetext{
${ }^{1}$ S. Sachdev, Quantum Phase Transitions (Cambridge University Press, Cambridge, UK, 1999).

${ }^{2}$ P. Coleman, C. Pépin, Q. Si, and R. Ramazashvili, J. Phys.: Condens. Matter 13, R723 (2001).

${ }^{3}$ Q. Si, S. Rabello, K. Ingersent, and J. L. Smith, Nature (London) 413, 804 (2001).

${ }^{4}$ A. Schröder, G. Aeppli, R. Coldea, M. Adams, O. Stockert, H. v. Löhneysen, E. Bucher, R. Ramazashvili, and P. Coleman, Nature (London) 407, 351 (2000).

${ }^{5}$ S. Friedemann, N. Oeschler, S. Wirth, C. Krellner, C. Geibel, F. Steglich, S. Paschen, S. Kirchner, and Q. Si, Proc. Natl. Acad. Sci. USA 107, 14547 (2010).

${ }^{6}$ Q. Si, S. Rabello, K. Ingersent, and J. L. Smith, Phys. Rev. B 68 , 115103 (2003).

${ }^{7}$ Q. Si and J.L. Smith, Phys. Rev. Lett. 77, 3391 (1996).

${ }^{8}$ J. L. Smith and Q. Si, Europhys. Lett. 45, 228 (1999); arXiv:cond-mat/9705140.

${ }^{9}$ A. M. Sengupta, Phys. Rev. B 61, 4041 (2000); arXiv:cond-mat/9707316.

${ }^{10}$ Q. Si, J. L. Smith, and K. Ingersent, Int. J. Mod. Phys. B 13, 2331 (1999).

${ }^{11}$ L. Zhu and Q. Si, Phys. Rev. B 66, 024426 (2002).

${ }^{12}$ G. Zaránd and E. Demler, Phys. Rev. B 66, 024427 (2002).

${ }^{13}$ M. T. Glossop and K. Ingersent, Phys. Rev. Lett. 95, 067202 (2005).
}

${ }^{14}$ M. T. Glossop and K. Ingersent, Phys. Rev. B 75, 104410 (2007).

${ }^{15}$ D. Withoff and E. Fradkin, Phys. Rev. Lett. 64, 1835 (1990).

${ }^{16}$ R. Bulla, Th. Pruschke, and A. C. Hewson, J. Phys.: Condens. Matter 9, 10463 (1997).

${ }^{17}$ C. Gonzalez-Buxton and K. Ingersent, Phys. Rev. B 57, 14254 (1998).

${ }^{18}$ K. Ingersent and Q. Si, Phys. Rev. Lett. 89, 076403 (2002).

${ }^{19}$ L. Fritz and M. Vojta, Phys. Rev. B 70, 214427 (2004).

${ }^{20}$ M. T. Glossop, S. Kirchner, J. H. Pixley and Q. Si, Phys. Rev. Lett. 107, 076404 (2011).

${ }^{21}$ M. Vojta and M. Kirćan, Phys. Rev. Lett. 90, 157203 (2003).

${ }^{22}$ M. Kirćan and M. Vojta, Phys. Rev. B 69, 174421 (2004).

${ }^{23}$ M. T. Glossop, N. Khoshkou and K. Ingersent, Phys. B (Amsterdam) 403, 1303 (2008).

${ }^{24}$ C. H. Chung, M. T. Glossop, L. Fritz, M. Kirćan, K. Ingersent, and M. Vojta, Phys. Rev. B 76, 235103 (2007).

${ }^{25}$ I. Affleck and A. W. W. Ludwig, Nucl. Phys B 360, 641 (1991).

${ }^{26}$ J. L. Cardy, Nucl. Phys. B 240, 514 (1984).

${ }^{27}$ P. Ginsparg, Fields, Strings and Critical Phenomena (Elsevier, New York, 1989).

${ }^{28}$ A. M. Tsvelik, Quantum Field Theory in Condensed Matter Physics (Cambridge University Press, Cambridge, UK, 1996).

${ }^{29}$ Throughout this paper, we adopt units where $\hbar=k_{B}=1$. 
${ }^{30}$ For $2 \lambda \geqslant 1$, the Fourier-transform integral does not converge, necessitating introduction of a finite cutoff scale that spoils $\omega / T$ scaling.

${ }^{31}$ S. Kirchner and Q. Si, Phys. Rev. Lett. 100, 026403 (2008).

${ }^{32}$ J. H. Pixley, S. Kirchner, K. Ingersent and Q. Si, Phys. Rev. Lett. 109, 086403 (2012).

${ }^{33}$ E. Gull, A. J. Millis, A. I. Lichtenstein, A. N. Rubtsov, M. Troyer, and P. Werner, Rev. Mod. Phys 83, 349 (2011).

${ }^{34}$ J. H. Pixley, S. Kirchner M. T. Glossop, and Q. Si, J. Phys.: Conf. Ser. 273, 012050 (2011).

${ }^{35}$ P. Werner and A. J. Millis, Phys. Rev. Lett. 99, 146404 (2007).

${ }^{36} \mathrm{P}$. Werner and A. J. Millis, Phys. Rev. Lett. 104, 146401 (2010).

${ }^{37}$ J. Otsuki, Phys. Rev. B 87, 125102 (2013).

${ }^{38}$ U. Weiss, Quantum Dissipative Systems,3rd ed., Series in Modern Condensed Matter Physics, Vol. 13 (World Scientific, Singapore, 2008).

${ }^{39}$ K. Binder, Z. Phys. B 43, 119 (1981).

${ }^{40}$ In problems that exhibit strict particle-hole symmetry, the U(1) symmetry that leads to conservation of total charge (electron number measured from half-filling) $Q_{\text {tot }}=\sum_{n, \sigma}\left(f_{n \sigma}^{\dagger} f_{n \sigma}-\frac{1}{2}\right)$ is promoted to an $\mathrm{SU}(2)$ isospin symmetry generated by $I_{\text {tot }}^{z} \equiv \frac{1}{2} Q_{\text {tot }}$ and $I_{\mathrm{tot}}^{+} \equiv\left(I_{\mathrm{tot}}^{-}\right)^{\dagger}=\sum_{n}(-1)^{n} f_{n \uparrow}^{\dagger} f_{n \downarrow}^{\dagger}$. The NRG calculations were performed on a basis of multiplets labeled by quantum numbers
$S_{\text {tot }}^{z}$ (the $z$ component of the total spin) and $I_{\text {tot }}$, with each multiplet representing $2 I_{\mathrm{tot}}+1$ degenerate eigenstates.

${ }^{41}$ M. Vojta, N.-H. Tong, and R. Bulla, Phys. Rev. Lett. 94, 070604 (2005).

${ }^{42}$ M. Vojta, R. Bulla, F. Guttge, and F. Anders, Phys. Rev. B 81, 075122 (2010).

${ }^{43}$ M. Vojta, Phys. Rev. B 85, 115113 (2012).

${ }^{44}$ S. Kirchner, Q. Si, and K. Ingersent, Phys. Rev. Lett. 102, 166405 (2009).

${ }^{45}$ S. Kirchner, J. Low Temp. Phys. 161, 282 (2010).

${ }^{46}$ S. Kirchner, K. Ingersent, and Q. Si, Phys. Rev. B 85, 075113 (2012).

${ }^{47}$ O. Parcollet, A. Georges, G. Kotliar and A. Sengupta, Phys. Rev. B 58, 3794 (1998).

${ }^{48}$ L. Zhu, S. Kirchner, Q. Si, and A. Georges, Phys. Rev. Lett. 93, 267201 (2004).

${ }^{49}$ F. Zamani, P. Ribeiro, J. H. Pixley, and S. Kirchner (unpublished). ${ }^{50}$ W. C. Oliveira and L. N. Oliveira, Phys. Rev. B 49, 11986 (1994).

${ }^{51}$ The strong-coupling spectrum of the particle-hole-symmetric pseudogap Anderson and Kondo models is characterized by a phase shift of $(1-r) \pi / 2$ for electrons at the Fermi energy (see Ref. 17).

${ }^{52}$ We thank M. Vojta for drawing this point to our attention.

${ }^{53}$ S. Kirchner, L. Zhu, Q. Si, and D. Natelson, Proc. Natl. Acad. Sci. USA 102, 18824 (2005). 